\title{
Aspects of the UV/IR correspondence: energy broadening and string fluctuations
}

\author{
Y. Hatta, ${ }^{a}$ E. Iancu, ${ }^{b, c}$ A.H. Mueller ${ }^{d}$ and D.N. Triantafyllopoulos ${ }^{e}$ \\ ${ }^{a}$ Graduate School of Pure and Applied Sciences, University of Tsukuba, \\ Tsukuba, Ibaraki 305-8571, Japan \\ ${ }^{b}$ CERN, Theory Division, \\ CH-1211 Geneva, Switzerland \\ ${ }^{c}$ Institut de Physique Théorique de Saclay, \\ F-91191 Gif-sur-Yvette, France \\ ${ }^{d}$ Department of Physics, Columbia University, \\ New York, NY 10027, U.S.A. \\ ${ }^{e}$ ECT*, Strada delle Tabarelle 286, \\ I-38123 Villazzano (TN), Italy \\ E-mail: hatta@het.ph.tsukuba.ac.jp, edmond.iancu@cea.fr, \\ amh@phys.columbia.edu, trianta@ect.it
}

ABSTRACT: We show that a source which radiates in the vacuum of the strongly coupled $\mathcal{N}=4$ SYM theory produces an energy distribution which, in the supergravity approximation, has the same space-time pattern as the corresponding classical distribution: the radiation propagates at the speed of light without broadening. We illustrate this on the basis of several examples: a small perturbation propagating down a steady string, a massless particle falling into $\mathrm{AdS}_{5}$, and the decay of a time-like wave-packet. A similar observation was made in Phys. Rev. D81 (2010) 126001 for the case of a rotating string. In all these cases, the absence of broadening is related to the fact that the energy backreaction on the boundary arises exclusively from the bulk perturbation at, or near, the boundary. This is so since bulk sources which propagate in $\mathrm{AdS}_{5}$ at the speed of light do not generate any energy on the boundary. We interpret these features as an artifact of the supergravity approximation, which fails to encode quantum mechanical fluctuations that should be present even in the strong coupling limit. We argue that such fluctuations should enter the dual string theory as longitudinal string fluctuations, which are not suppressed at strong coupling. We heuristically estimate the effects of such fluctuations and argue that they restore the broadening of the radiation, in agreement with expectations from both quantum mechanics and the ultraviolet/infrared correspondence.

KEYwords: AdS-CFT Correspondence, Strong Coupling Expansion

ARXIV EPRINT: 1011.3763 


\section{Contents}

1 Introduction $\quad 1$

2 Classical strings $\quad 6$

$\begin{array}{lll}2.1 & \text { Heavy quark with constant velocity } & 7\end{array}$

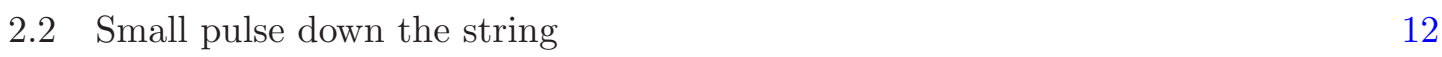

$\begin{array}{ll}2.3 \text { Velocity of a bit of energy } & 18\end{array}$

3 A time-like wave-packet $\quad 22$

3.1 The falling massless particle 24

$\begin{array}{ll}3.2 & \text { A time-like wave-packet } 26\end{array}$

4 String fluctuations and the freely falling particle $\quad 29$

4.1 A special set of light-cone coordinates 30

4.2 Longitudinal string fluctuations and broadening 32

$\begin{array}{ll}\text { A The constant velocity string: a different perspective } & 37\end{array}$

$\begin{array}{ll}\text { B Boundary to bulk scalar propagator in } \mathrm{AdS}_{5} & 38\end{array}$

$\begin{array}{ll}\text { C Backreaction in the non-relativistic limit } & 39\end{array}$

$\begin{array}{ll}\text { D Strings in the light-cone gauge } & 41\end{array}$

\section{Introduction}

Understanding gauge theory dynamics beyond perturbation theory, and in particular at strong coupling, represents one of the major desiderata of theoretical physics, with ramifications going from high-energy to condensed matter physics. Recent years have seen some important progress in that sense thanks to the theoretical breakthrough known as the AdS/CFT correspondence (or, with a somewhat more general sense, the gauge/string duality) [1-3], which allows one to study the strong coupling regime of some special, highly symmetric, gauge theories via weak coupling techniques in a 'dual' string theory. Further efforts in that direction have been triggered by the experimental observation of strong coupling aspects in the dynamics of the quark-gluon plasma - the high-temperature, deconfined, phase of QCD, which is produced in the intermediate stages of a heavy ion collision at RHIC and, more recently, at the LHC. This plasma corresponds to a physical regime where QCD itself is not so far away from its conformal 'cousins', so like the $\mathcal{N}=4$ supersymmetric Yang-Mills (SYM), to which the AdS/CFT correspondence has been widely 
tested. This observation stimulated applications of AdS/CFT to time-dependent phenomena (see, e.g., the review papers [4-6] and refs. therein), among which the problem of the radiation at strong coupling [7-21, 23, 24].

In particular, some of these studies addressed the space-time distribution of the radiated energy, whose calculation in AdS/CFT is quite complex, as it requires solving a gravitational backreaction problem (see below). Motivated by the phenomenology of the quark-gluon plasma, the first such studies were concerned with the finite-temperature problem - e.g., the medium-induced radiation by a heavy quark propagating through a strongly coupled plasma [13, 14, 17-19]. More recently, similar problems have been addressed also in the context of the vacuum of the $\mathcal{N}=4 \mathrm{SYM}$ theory $[15,16,20,22,23]$. One of these studies - that of the synchrotron radiation in ref. [23] — produced some very interesting and intriguing results, which in particular triggered our present analysis.

Before we describe these results and our present work, let us recall that all the AdS/CFT calculations aforementioned have been performed in the supergravity approximation (SUGRA), which is the semiclassical limit of the string theory in which both string loops and internal string excitations are neglected. This approximation is generally assumed to faithfully describe the strong 't Hooft coupling limit of the $\mathcal{N}=4$ SYM theory, that is, the limit $\lambda=g^{2} N_{c} \rightarrow \infty$ with fixed $g \ll 1$ ( $g$ is the Yang-Mills coupling and $N_{c}$ the number of colors). However, in this work we shall give arguments suggesting that this is not always the case: SUGRA seems unable to capture the detailed space-time distribution of the radiation emitted in the vacuum and in the strong coupling limit. This is so since, as we shall demonstrate, this distribution is affected by longitudinal string fluctuations which are not suppressed in the strong coupling limit.

Our arguments in that sense will be constructed in two steps, with different degrees of rigor: (1) a supergravity calculation of the energy backreaction in sections 2 and 3 , which is very explicit and in some cases even exact, and (2) a calculation of the string fluctuations in section 4, which is semi-heuristic (in the sense of using string quantization in flat spacetime), but which we believe to capture the salient features of the actual situation in curved space-time. In what follows, we shall describe these two steps in more detail.

The first step involves explicit calculations of the energy distribution produced in the supergravity approximation by various types of sources radiating in the vacuum of $\mathcal{N}=4$ SYM. One purpose of this analysis is to demonstrate that the main result in ref. [23] is in fact generic (and not specific, say, to the special geometry of the rotating quark). Namely, the supergravity prediction for the radiation emitted by an arbitrary source exhibits the same space-time pattern as the corresponding classical result, without any trace of broadening. By 'broadening' we mean off-shell effects, which would be natural in a quantum field theory (and even more so at strong coupling !) and would yield components of the radiation which propagate slower than light, thus leading to a spreading in the radiation emitted by a source which is localized in space and time. But our calculations show that, in the SUGRA limit, the radiation always propagates at the speed of light, so like the solution to the classical Maxwell equations. For instance, a point-like source at $r=0$ which is a pulse in time with duration $\sigma$ generates a thin spherical shell of energy, which is localized at $r=t$ with a width equal to $\sigma$. 
One reason why this lack of broadening looks so surprising is because, at a first sight at least, it seems to contradict the ultraviolet/infrared (UV/IR) correspondence [25, 26], which is one of the pillars of the gauge/string duality. In order to explain this puzzle, let us first recall some elements of the AdS/CFT formalism and thus fix some notations.

The five-dimensional (5D) Anti de Sitter space-time $\mathrm{AdS}_{5}$ where the string theory is formulated can be viewed as a product between the $4 \mathrm{D}$ Minkowski space-time (the 'boundary' of $\mathrm{AdS}_{5}$ ) times a 'radial' (or 'fifth') dimension, that we shall denote as $z$, in conventions where $0 \leq z<\infty$ and the Minkowski boundary lies at $z=0$ (see eq. (2.1) for the precise form of our metric). Roughly speaking, this fifth dimension acts as a reservoir of quantum fluctuations for the dual gauge theory. The UV/IR correspondence is a more precise version of this statement. This is a unique tool allowing for the physical interpretation of the results of the string theory back to the original gauge theory.

Specifically, the UV/IR correspondence relates the typical energy/momentum scales (or, by the uncertainty principle, the space-time scales) of the physical phenomenon on the 'boundary' to the radial $(z)$ position of the string excitation in the 'bulk'. For real-time phenomena like radiation, there are at least two types of scales which can be encoded in this way: (i) the overall size $R$ of the phenomenon on the boundary, ${ }^{2}$ which with our present conventions is proportional to the radial penetration $z$ of the dual excitation in the bulk, and (ii) the space-time virtuality $\Delta s^{2}$ on the boundary (say, the difference $t^{2}-r^{2}$ in the case of the spherical shell of radiation alluded to above), which is proportional to the position $z$ of the string source in the bulk at the time of emission. For the physical problems that we have in mind and that we shall discuss in this paper, the above statements are direct consequences of causality within the framework of the string theory calculation of the backreaction. This is the calculation of the energy density on the boundary from the response of the $\mathrm{AdS}_{5}$ metric to the $5 \mathrm{D}$ stress tensor of the string excitation (which for this purpose plays the role of a 'source').

How do these scaling arguments apply to the supergravity results for radiation at strong coupling? First of all, we should say that they work fine for the radiation emitted in a medium $[13,14,18,19]$. For instance, a heavy quark moving at constant speed in the $\mathcal{N}=4$ SYM plasma at temperature $T$ is dual to the 'trailing string' — a Nambu-Goto string attached to the quark and pulled by the latter through the $\mathrm{AdS}_{5}$ black hole geometry representing the plasma. Each bit of this string, which in the 5th direction extends from the Minkowski boundary down to the black hole horizon at $z \sim 1 / T$, yields a contribution to the energy on the boundary with a transverse width proportional to the location $z$ of that bit $[13,14,19]$. In particular, when $z$ (and hence the transverse width) becomes of order $1 / T$, this is a sign that the respective component of the radiation has thermalized (and then propagates as a hydrodynamical wave in the medium [14]).

Returning to the problem of the radiation in the vacuum, we note that the first 'UV/IR correlation' above - that between the overall size and the radial penetration of the dual

\footnotetext{
${ }^{1}$ Following standard conventions, we shall use the word 'radial' in relation with both the 5 th dimension $z$, and the physical radius $r=|\boldsymbol{r}|$ in 3 dimensions. The precise meaning should be clear from the context.

${ }^{2}$ Here $R$ refers to a frame where the center of mass of the energy distribution is at rest; in other frames, one must take into account the Lorentz contraction (see e.g. the discussion in section 2.1).
} 
excitation in the bulk - is indeed respected, as is easy to see for a localized excitation. For instance, for the 'pulse on the boundary' alluded to above (more precisely, a time-like wavepacket which can represent the virtual photon produced in electron-positron annihilation), we shall find that the dual bulk perturbation propagates like a massless particle falling into $\mathrm{AdS}_{5}$ (a wave-packet centered at $z=t$ ). This is in agreement with the fact that, on the boundary, the energy density expands as a spherical shell at $r=t$ (see the discussion in sections 3.1 and 3.2). A similar situation holds when the physical source is a heavy quark whose dual object in the bulk is a Nambu-Goto string. Then, the bulk perturbation which propagates at the 5D speed of light is a bit of energy flowing down the string. (The calculation of this $5 \mathrm{D}$ velocity requires a careful separation of the radiative part from the Coulomb part of the energy, which are both encoded in the Nambu-Goto string; see section 2.3 and the subsequent publication [27] for more details.) This is in agreement with the fact that the radiation produced via backreaction on the boundary expands outward at the speed of light, as found in [23] for the rotating string and as we shall see in section 2.2 below for a small but generic string perturbation.

What about the other 'UV/IR correlation', that between the space-time virtuality of the radiation on the boundary and the position $z$ of its source in the bulk? At first sight, this seems to be violated by the SUGRA results that we are aware of: the radiation propagates in the vacuum without broadening, so like massless, on-shell, quanta, in spite of the fact that there are sources in the bulk at arbitrary large distances $z$. Such a violation would be very strange though: as we shall argue in section 2.2 , the correlation between the broadening of the distribution on the boundary and the position of the source in the bulk is simply a consequence of causality. (Such a correlation is for instance manifest in the calculation of the 'glueball' density $\operatorname{Tr} F_{\mu \nu}^{2}$ produced on the boundary by a small perturbation of a static string [28].) And indeed a closer inspection of the calculations in sections 2 and 3 and also in ref. [23] reveals that this apparent contradiction is solved by the fact that, in all these calculations, the whole backreaction on the boundary (in terms of radiation) comes from sources in the bulk which are located close to the boundary, within a distance in $z$ set by the width $\sigma$ of the physical perturbation. In particular, in the limit where the physical source has zero width, the backreaction on the boundary comes exclusively from the limit $z \rightarrow 0$ of the perturbation in the bulk. ${ }^{3}$

But whereas it solves a potential conflict with the UV/IR correspondence, this last observation introduces another puzzle: why should bulk sources which lie far away from the boundary have no reflection in terms of energy density in the physical gauge theory ? Our investigations will allow us to elucidate the mathematical origin of this mysterious property in the context of the SUGRA calculations: it arises from the fact that bulk sources which propagate in $\mathrm{AdS}_{5}$ at the speed of light do not generate any energy backreaction on the boundary. ${ }^{4}$ This property is quite intuitive when the source is moving parallel to the

\footnotetext{
${ }^{3}$ In fact, even in more general cases where the physical source is delocalized on the boundary (like the rotating quark in ref. [23]) it appears that, via appropriate changes of variables in the convolutions expressing the backreaction, one can obtain the whole result for the energy density as an endpoint contribution, coming from the boundary endpoint $(z=0)$ of the integral over $z[23,27]$.

${ }^{4}$ The importance of the bulk propagation at the speed of light for the problem of the synchrotron
} 
boundary, that is, it stays at a fixed value of the 5th coordinate $z$ : then, the backreaction is a gravitational shock-wave [30] which, because of Lorentz time dilation, is fully generated at very early times $t \rightarrow-\infty$ (see section 2.1 below). In that case, there is naturally no energy backreaction from any finite value of $t$. Similarly, for the case of a massless particle falling into $\mathrm{AdS}_{5}$ at the speed of light (say, along the 5th dimension), we shall find that there is no energy backreaction except from the starting point of the trajectory at $t=z=0$. The corresponding backreaction is a gravitational shock-wave with zero width (since fully generated at $z=0$ ) and whose intersection with the boundary is a spherical shell moving outward with $r=t$.

For motion at the speed of light which is oriented towards the interior of $\mathrm{AdS}_{5}$, one can formally interpret the absence of energy backreaction as a kind of 'Lorentz time dilation', but only after performing a special change of coordinates which mixes the fifth dimension with one of the spatial dimensions (see section 4.1). In these new coordinates, the propagation of the radiation without broadening appears as 'Lorentz contraction'. But we do not find this interpretation as very natural, or useful.

More generally, we believe that there is no physical foundation for this property of the supergravity approximation, which is the lack of broadening for radiation at strong coupling. Indeed, this seems difficult to reconcile with general expectations about the dynamics in an interacting quantum field theory, like the importance of off-shell effects generated by virtual quantum fluctuations. In particular, this property is inconsistent with a physical picture for parton branching at strong coupling [16], which is supported by other AdS/CFT calculations, like those concerning the anomalous dimensions for the leading-twist operators [31-34], the structure functions for deep inelastic scattering [3538 ], or the angular distribution of the energy density produced via the decay of a time-like wave-packet [15]. Note however that none of these previous calculations has investigated the detailed space-time distribution of the energy density, so they could not encounter the difficulties that we are concerned with here.

Motivated by such considerations which make us feel uncomfortable with the supergravity prediction for the energy distribution, we shall proceed in section 4 to a study of the stability of this approximation with respect to string fluctuations. Such a study is necessarily heuristic since the quantization of the string fluctuations in curved space-time is an unsolved problem. Here, we shall follow a pragmatic approach proposed by Hofman and Maldacena [15], which consists in using string quantization in flat space and the light-cone (LC) gauge together with a special change of coordinates, introduced in [39], which mixes the fifth coordinate $z$ with one of the spatial coordinates (see section 4.1). Hofman and Maldacena used this strategy to study the angular distribution of the energy produced by

radiation in AdS/CFT has been also recognized in the recent paper [29]. There it is shown that each energy bit flowing down the rotating string generates a backreaction in the form of a gravitational shock-wave and that, by superposing such shock-waves for all the bits along the string, one obtains a pattern for the energy density on the boundary which is similar to that found in [23]. However, it was not realized there that each of these shock-waves is in fact generated via backreaction from $z \approx 0$ (that is, from the very early stages of the trajectory of each energy bit) and that this is the reason why the resulting energy distribution shows no more broadening than the classical one. 
the decay of a time-like wave-packet. They computed only the energy density integrated over the spatial radius $r$, so they could not notice that the energy is actually localized on a thin shell at $r=t$. For that particular set-up, they have shown that the energy distribution in the SUGRA approximation is isotropic event by event and that the string corrections to it - which involve the transverse string fluctuations - are suppressed, as expected, in the strong coupling limit.

Here, however, we shall be concerned with the radial $(r)$ distribution of the energy density, which in the coordinates of ref. [39] receives corrections from the longitudinal $l^{5}$ string fluctuations. The latter are not independent degrees of freedom, rather they are related to the transverse fluctuations via the constraints of the LC gauge, but this relation is such that the longitudinal fluctuations are not suppressed as $\lambda \rightarrow \infty$. This last property is well known (it is manifest in textbook treatments of string quantization in flat space $[40,41]$ ), yet we are not aware of any application of it in the context of AdS/CFT. This is most likely so because the longitudinal degrees of freedom do not directly participate in standard string problems like the scattering between two strings.

Following the strategy in [15], in section 4.2 we shall show that the radial distribution of the energy density receive sizeable corrections from the longitudinal string fluctuations. These corrections are independent of $\lambda$ - at least up to issues of ultraviolet divergences, that we shall shortly comment on. Hence they are a part of the leading order result in the strong coupling limit and they act, as expected, towards spreading the distribution in $r-t$. Moreover this spreading is such that the UV/IR correspondence is respected: a contribution to the energy density from string fluctuations at $z$ yields a spreading $t-r \sim z$.

Our present analysis cannot be seen as definitive, first, because of the lack of rigor in the string quantization prescription and, second, because the analysis in section 4.2 is plagued with ultraviolet divergences, as is generally the case for problems involving string fluctuations in flat space. Polchinski and Susskind have argued that, in $\mathrm{AdS}_{5}$ the UV divergences should be cured by the warp factor [42]. We do not know whether the arguments in ref. [42] can be extended to the longitudinal fluctuations of interest to us here. But independently of such issues, which require further clarifications, we believe that our results provide solid evidence in favor of the failure of the supergravity approximation for the observables which are sensitive to longitudinal string fluctuations.

\section{Classical strings}

In this section, we shall present our first example of a supergravity calculation of radiation at strong coupling, whose result shows the remarkable feature outlined in the Introduction: the radiated energy propagates without broadening, in spite of having bulk sources at arbitrarily large distances in the 5th dimension.

Specifically, in section 2.2 we shall compute the energy density radiated by a small perturbation ('pulse') propagating along a steady string. In physical terms, the string describes the wavefunction of a heavy quark and the pulse represents its response to an external perturbation acting for a short lapse of time. The condition that the string

\footnotetext{
${ }^{5}$ By which we mean the space-time direction longitudinal in the sense of the LC gauge; see section 4.
} 
perturbation remain small is equivalent to the non-relativistic approximation for the motion of the heavy quark. We shall find that the whole contribution to radiation (as obtained after subtracting the Coulomb piece of the energy) is generated via backreaction from the string endpoint at the boundary alone. Via the UV/IR correspondence, this property is correlated with the lack of broadening alluded to above.

But before turning to the string perturbation, we shall first review, in section 2.1, the calculation of the energy backreaction for an unperturbed string moving at constant speed. Besides allowing us to introduce the general formalism in a simpler setup, this problem is interesting also in that it offers a conceptually simple example where the UV/IR correspondence works as expected - in relation with the Coulomb energy of the heavy quark. Moreover, in this context we shall for the first time observe a property which will play an important role later on: a bulk source which propagates at the $5 \mathrm{D}$ speed of light does not generate any energy on the Minkowski boundary.

Finally in section 2.3 we shall argue that this last property is responsible for the lack of backreaction from string points away from the boundary, for both the small string perturbation studied in section 2.2 and the rotating string problem discussed in ref. [23].

\subsection{Heavy quark with constant velocity}

Consider an on-shell heavy quark moving with constant velocity $v<1$ along the $x \equiv x^{1}$ axis within the vacuum of the $\mathcal{N}=4$ SYM theory. In the dual string theory, and with a convenient choice of coordinates in $\mathrm{AdS}_{5}$, this is described as a Nambu-Goto string attached to a D7-brane hanging vertically along the radial direction of $\mathrm{AdS}_{5}$ and moving at constant speed $v^{M}=v \delta^{M 1}$. ( $M=0,1,2,3,5$ are vector indices for $\mathrm{AdS}_{5}$ : the first four indices refer to the Minkowski components, and the 5th one to the radial direction.) Specifically, using the so-called Poincaré coordinates,

$$
\mathrm{d} s^{2} \equiv G_{M N} \mathrm{~d} x^{M} \mathrm{~d} x^{N}=\frac{L^{2}}{z^{2}}\left[-\mathrm{d} t^{2}+\mathrm{d} \boldsymbol{r}^{2}+\mathrm{d} z^{2}\right]
$$

together with the temporal-gauge parametrization $X^{M}(\tau, \sigma) \equiv X^{M}(t, z)$ for the string world-sheet, the string embedding function reads $X^{M}=(t, v t, 0,0, z)$. In eq. (2.1), $\boldsymbol{r}=$ $\left(x^{1}, x^{2}, x^{3}\right)$ and we shall often use the notations $x \equiv x^{1}$ and $\boldsymbol{x}_{\perp} \equiv\left(x^{2}, x^{3}\right)$. The variable $z$ along the string is restricted to $z \geq z_{m}$ where $z_{m}=\sqrt{\lambda} /\left(2 \pi m_{q}\right)$ is the radial position of the D7-brane and $m_{q}$ is the mass of the heavy quark. In what follows we shall assume the quark to be heavy enough for $z_{m}$ to be much smaller than all the other scales in the problem (e.g., the space-time locations where we measure the energy density), and we shall often set $z_{m}=0$ in explicit calculations.

The moving quark generates (color) electric and magnetic fields, which are simply the boosted versions of the Coulomb field produced by the quark in its rest frame. We would like to compute the energy density $\mathcal{E} \equiv\left\langle T_{00}\right\rangle$ which is stored in these fields. Within the supergravity context, this is obtained from the 'backreaction' of the associated NambuGoto string on the Minkowski boundary [43, 44]. The respective construction is in fact more general than the specific quark/string problem at hand - it applies whenever we 
have a 'source' of energy and momentum in the bulk, with $5 \mathrm{D}$ stress tensor $t_{M N}$, which is dual to some physical perturbation, or bound state, in the boundary gauge theory.

In what follows, we shall exhibit the general formulæ expressing the energy backreaction for the type of problems of interest to us here - namely, for bulk sources which are dual to small perturbations of the $\mathcal{N}=4 \mathrm{SYM}$ vacuum. (This is in particular the case for the heavy quark problem under consideration.) By 'small perturbations', we more precisely mean sources $t_{M N}$ which in the limit $N_{c} \rightarrow \infty$ scale like $N_{c}^{0}=1$, so the associated change $h_{M N} \equiv \delta G_{M N}$ in the bulk metric is a small effect of $\mathcal{O}\left(1 / N_{c}^{2}\right)$. To compute this effect, it is enough to solve the linearized version of the 5D Einstein equations. The gauge theory stress tensor $\left\langle T_{\mu \nu}\right\rangle$ is finally inferred from a study of the behaviour of the metric perturbation $h_{M N}$ near the Minkowski boundary at $z=0$. By working in a gauge where $h_{5 M}=0$ and denoting

$$
H_{\mu \nu} \equiv \frac{z^{2}}{L^{2}} h_{\mu \nu}=H_{\mu \nu}^{(3)} z^{3}+H_{\mu \nu}^{(4)} z^{4}+\mathcal{O}\left(z^{5}\right),
$$

one can compute the gauge theory expectation value of the stress tensor from the coefficient of $z^{4}$ in the above small $-z$ expansion:

$$
T_{\mu \nu}=\frac{2 L^{3}}{\kappa_{5}^{2}} H_{\mu \nu}^{(4)}=\frac{N_{c}^{2}}{2 \pi^{2}} H_{\mu \nu}^{(4)} .
$$

Although it amounts to solving linearized equations of motion, the calculation of $H_{M N}$ is quite involved, because of the many coupled equations for the (generally) fifteen independent components. However, this calculation can be more economically organized by recognizing that the fifteen 'independent' components of $H_{M N}$ depend upon only five gauge-invariant degrees of freedom, in agreement with the number of physical degrees of freedom expected for the $4 \mathrm{D}$ stress tensor $T_{\mu \nu}$. These physical degrees of freedom (linear combinations of $H_{M N}$ and its derivatives) can be chosen in (arbitrarily) many ways, and here we shall follow the procedure in [23], from which we shall simply quote the relevant results (see refs. $[14,45]$ for other such choices).

Namely, specializing to $\mathcal{E} \equiv T_{00}$ and using eq. (3.62) in ref. [23], one finally arrives at the following expression for the energy density at a space-time point $x^{\mu}=(t, \boldsymbol{r})$ on the boundary:

$$
\mathcal{E}(t, \boldsymbol{r})=\mathcal{E}_{\mathrm{A}}(t, \boldsymbol{r})+\mathcal{E}_{\mathrm{B}}(t, \boldsymbol{r})
$$

where

$$
\begin{aligned}
& \mathcal{E}_{\mathrm{A}}=\frac{2 L^{3}}{\pi} \int \frac{\mathrm{d}^{4} \dot{r} \mathrm{~d} z}{z^{2}} \Theta(t-\hat{t}) \delta^{\prime \prime}(\mathcal{W})\left[z\left(2 t_{00}-t_{55}\right)-(t-\hat{t}) t_{05}+(x-\dot{x})^{i} t_{i 5}\right] \\
& \mathcal{E}_{\mathrm{B}}=\frac{2 L^{3}}{3 \pi} \int \frac{\mathrm{d}^{4} \dot{r} \mathrm{~d} z}{z} \Theta(t-\hat{t}) \delta^{\prime \prime \prime}(\mathcal{W})\left[|\boldsymbol{r}-\dot{\boldsymbol{r}}|^{2}\left(2 t_{00}-2 t_{55}+t_{i i}\right)-3(x-\dot{x})^{i}(x-\hat{x})^{j} t_{i j}\right] .
\end{aligned}
$$

In these equations, the bulk point with coordinates $(\dot{t}, \dot{\boldsymbol{r}}, z)$ is the source point from which originates the perturbation and $\mathrm{d}^{4} \dot{r} \equiv \mathrm{d} \dot{t} \mathrm{~d}^{3} \dot{r}$. (Throughout this paper, we shall systematically use the symbol acute for the spatial coordinates of a point belonging to a source in 
the bulk.) Furthermore, the quantity

$$
\mathcal{W}=-(t-\hat{t})^{2}+(\boldsymbol{r}-\dot{\boldsymbol{r}})^{2}+z^{2} .
$$

is proportional to the $5 \mathrm{D}$ invariant distance between that source point in the bulk and the point of measurement on the boundary. The $\delta$-function $\delta(\mathcal{W})$, which enters via the (retarded) bulk-to-boundary propagator [23], shows that the metric perturbation propagates throughout $\mathrm{AdS}_{5}$ at the $5 \mathrm{D}$ speed of light (which is equal to one in our present conventions), as expected for classical, massless fields in $\mathrm{AdS}_{5}$ - in this case, gravitational waves. The presence of derivatives of the $\delta$-function may seem peculiar, but it is a generic feature of retarded propagators in AdS, as we shall show in appendix B where we construct the respective scalar propagator. The derivatives of $\delta(\mathcal{W})$ in eqs. $(2.5)$ and $(2.6)$ can be taken as derivatives w.r.t. one of the external variables appearing in $\mathcal{W}$ and subsequently pulled out of the integrand, but caution is needed since there might be explicit dependence on that variable in the integrand. In such cases it is better to write

$$
\delta^{(n)}(\mathcal{W})=\lim _{\epsilon \rightarrow 0} \partial_{\epsilon}^{n} \delta(\mathcal{W}+\epsilon)
$$

As already emphasized, eqs. (2.5) and (2.6) hold for an arbitrary bulk source with (parametrically small) stress tensor $t_{M N}$. When this source is a string - the case of interest in this section - , there are some simplifications due to the fact that, for a string, $t_{M N}$ has support only on the 2D string world-sheet. Using the parametrization $X^{M}=\left(t, \boldsymbol{r}_{s}(t, z), z\right)$ for the latter, one has $t_{M N}(\dot{t}, \dot{\boldsymbol{r}}, z) \propto \delta^{(3)}\left(\dot{\boldsymbol{r}}-\boldsymbol{r}_{s}(\dot{t}, z)\right)$ and then the spatial integrations in eqs. (2.5) and (2.6) are trivially done.

More explicitly, for a generic string profile $X^{M}=\left(t, \boldsymbol{r}_{s}(t, z), z\right)$, one has

$$
t^{M N}(t, \boldsymbol{r}, z)=-\frac{T_{0}}{\sqrt{-G}} \sqrt{-g} g^{a b} \partial_{a} X^{M} \partial_{b} X^{N} \delta^{(3)}\left(\boldsymbol{r}-\boldsymbol{r}_{s}\right) .
$$

where $T_{0}=\sqrt{\lambda} / 2 \pi L^{2}$ is the string tension, $G_{M N}$ is the (unperturbed) $\mathrm{AdS}_{5}$ metric from eq. (2.1), and $g_{a b}$, with $a, b=t, z$, is the induced metric on the string world-sheet:

$$
g_{a b}=G_{M N} \partial_{a} X^{M} \partial_{b} X^{N}, \quad-g=\left(\partial_{t} X \cdot \partial_{z} X\right)^{2}-\left(\partial_{t} X\right)^{2}\left(\partial_{z} X\right)^{2} .
$$

In what follows, we shall often use a dot (prime) to denote a derivative w.r.t. $t(z)$.

Armed with such general formulæ, we now return to the case of a heavy quark at constant velocity, for which we have $X^{M}=(t, v t, 0,0, z)$. The respective 5D bulk stress energy tensor is easily obtained as

$$
t_{M N}=\frac{\sqrt{\lambda}}{2 \pi} \frac{\gamma z}{L^{3}} \delta^{(2)}\left(\boldsymbol{x}_{\perp}\right) \delta(x-v t)\left(\begin{array}{ccc}
1 & -v & 0 \\
-v & v^{2} & 0 \\
0 & 0 & v^{2}-1
\end{array}\right)
$$

where, with increasing order, the elements correspond to the $t, x$ and $z$-components, while all the elements which involve at least one transverse component are identically zero and are not shown. $\gamma=1 / \sqrt{1-v^{2}}$ is the Lorentz contraction factor. Using eq. (2.5) we find

$$
\mathcal{E}_{\mathrm{A}}=\frac{\sqrt{\lambda}}{\pi^{2}} \gamma\left(3-v^{2}\right) \partial_{\boldsymbol{x}_{\perp}^{2}}^{2} \int \mathrm{d} z \mathrm{~d} \dot{t} \delta(\mathcal{W})
$$


where for the situation at hand

$$
\mathcal{W}=-(t-\hat{t})^{2}+(x-v \hat{t})^{2}+\boldsymbol{x}_{\perp}^{2}+z^{2} .
$$

Solving the constraint $\mathcal{W}=0$ and taking into account only the retarded solution, we write

$$
\delta(\mathcal{W})=\frac{\gamma \delta\left[\hat{t}-\gamma^{2}(t-v x)+\gamma \sqrt{\boldsymbol{x}_{\perp}^{2}+z^{2}+\gamma^{2}(x-v t)^{2}}\right]}{2 \sqrt{\boldsymbol{x}_{\perp}^{2}+z^{2}+\gamma^{2}(x-v t)^{2}}} .
$$

Integrating over $\dot{t}$ and taking the two derivatives we obtain

$$
\mathcal{E}_{\mathrm{A}}=\frac{3 \sqrt{\lambda}}{8 \pi^{2}} \gamma^{2}\left(3-v^{2}\right) \int \frac{\mathrm{d} z}{\left[\boldsymbol{x}_{\perp}^{2}+z^{2}+\gamma^{2}(x-v t)^{2}\right]^{5 / 2}} .
$$

The integral over $z$ can be easily performed to yield

$$
\mathcal{E}_{\mathrm{A}}=\frac{\sqrt{\lambda}}{4 \pi^{2}} \frac{\gamma^{2}\left(3-v^{2}\right)}{\left[\boldsymbol{x}_{\perp}^{2}+\gamma^{2}(x-v t)^{2}\right]^{2}} .
$$

Here, however, we are less interested in the final result for the energy density on the boundary, but more in the way how this result gets built by adding contributions coming from different points $z$ inside the bulk. We shall return to this issue in a moment, but before that let us also compute the second term $\mathcal{E}_{\mathrm{B}}$. Starting from eq. (2.6) we have

$$
\mathcal{E}_{\mathrm{B}}=\frac{\sqrt{\lambda} \gamma}{3 \pi^{2}} \lim _{\epsilon \rightarrow 0} \partial_{\epsilon}^{3} \int \mathrm{d} z \mathrm{~d} \dot{t} \delta(\mathcal{W}+\epsilon)\left[\left(4-v^{2}\right) \boldsymbol{x}_{\perp}^{2}+4\left(1-v^{2}\right)\left(x-v \hat{t}^{2}\right] .\right.
$$

It is straightforward to integrate over $t$ using the $\delta$-function, differentiate three times w.r.t. $\epsilon$, set $\epsilon=0$ and finally integrate over $z$ to arrive at

$$
\mathcal{E}_{\mathrm{B}}=-\frac{\sqrt{\lambda} \gamma^{2}}{6 \pi^{2}} \frac{\left(4-2 v^{2}\right)\left[\boldsymbol{x}_{\perp}^{2}+\gamma^{2}(x-v t)^{2}\right]+v^{2} \gamma^{2}(x-v t)^{2}}{\left[\boldsymbol{x}_{\perp}^{2}+\gamma^{2}(x-v t)^{2}\right]^{3}} .
$$

Adding the two contributions we obtain the expected result $[13,14,19]$

$$
\mathcal{E}=\frac{\sqrt{\lambda} \gamma^{2}}{12 \pi^{2}} \frac{\left(1+v^{2}\right) \boldsymbol{x}_{\perp}^{2}+(x-v t)^{2}}{\left[\boldsymbol{x}_{\perp}^{2}+\gamma^{2}(x-v t)^{2}\right]^{3}} .
$$

When $v \rightarrow 1$ this formula approaches a shock-wave, as expected from Lorentz contraction:

$$
\mathcal{E}=\frac{\sqrt{\lambda} \gamma}{16 \pi \boldsymbol{x}_{\perp}^{3}} \delta(x-t)
$$

This is in agreement with the shock-wave constructed in $[30,46]$ when the latter is integrated over all source positions $z_{*}$ in the bulk with a weight proportional to $1 / z_{*}^{2}$, as suggested from the expression of the static energy of a string hanging down.

We now turn to our main interest in this calculation, which is to show that the UV/IR correspondence is indeed satisfied in this case, in a rather precise, quantitative way. Specifically, a bit of the string located at radial distance $z$ from the boundary contributes predominantly to the Coulomb energy density around the space-time points $x^{\mu}=\left(t, x, \boldsymbol{x}_{\perp}\right)$ 
with $x_{\perp} \sim \gamma(x-v t) \sim z$ (here, $\left.x_{\perp}=\left|x_{\perp}\right|\right)$. This is simply the Lorentz boosted version of the (perhaps more familiar) statement that, in the rest frame of the heavy quark $(v=0)$, a bit of the string at $z$ is responsible for the backreaction at points $\boldsymbol{r}$ with $r \equiv|\boldsymbol{r}| \sim z$. This follows by inspection of the convergence properties of the integral over $z$ in eq. (2.15) and the corresponding one for $\mathcal{E}_{\mathrm{B}}$.

Consider eq. (2.15) for definiteness and assume that $x_{\perp} \sim \gamma(x-v t)$ and they are both very large as compared to the lower limit $z_{m}$ in the integral over $z$ (which has been kept implicit in equations like (2.12)). We can divide the integration range into three domains: (i) $z \ll x_{\perp}$, (ii) $z \simeq x_{\perp}$, and (iii) $z \gg x_{\perp}$. In domain (i), we can neglect $z$ within the integrand and the ensuing integral, that is,

$$
I_{1} \approx \int_{z_{m}}^{x_{\perp}} \frac{\mathrm{d} z}{\left[\boldsymbol{x}_{\perp}^{2}+\gamma^{2}(x-v t)^{2}\right]^{5 / 2}} \approx \frac{x_{\perp}}{\left[\boldsymbol{x}_{\perp}^{2}+\gamma^{2}(x-v t)^{2}\right]^{5 / 2}} \sim \frac{1}{x_{\perp}^{4}}
$$

is dominated by its upper limit $z \simeq x_{\perp}$ (at least parametrically and for relatively large values of $x_{\perp}$ ). In domain (iii), the external coordinates can be neglected and then

$$
I_{3} \approx \int_{x_{\perp}}^{\infty} \frac{\mathrm{d} z}{z^{5}} \sim \frac{1}{x_{\perp}^{4}}
$$

where this time the integral is dominated by its lower limit, that is, $z \simeq x_{\perp}$ once again. Thus, the integral is indeed controlled by values $z \sim x_{\perp}$. This is the expected manifestation of the UV/IR correspondence for the problem at hand. Note also the way how this correspondence works in the presence of a Lorentz boost: the longitudinal extent $x-v t$ of the energy generated by a bit of string at $z$ scales like $x-v t \sim z / \gamma$; this is the Lorentz-contracted version of the corresponding extent $x_{\perp} \sim z$ in transverse directions.

The second issue of interest to us here is the typical value of the 'emission' time $t$ which contributes to the backreaction. We would like to show that, in the ultrarelativistic limit $v \rightarrow 1$, or $\gamma \rightarrow \infty$, the whole contribution to the energy density - which in that limit takes the form of the shock-wave (2.20) - comes from very early times $t \rightarrow-\infty$. The corollary of that is that a bit of the string which propagates through $\mathrm{AdS}_{5}$ at the $5 \mathrm{D}$ speed of light ${ }^{6}$ does not produce any backreaction on the boundary (since there is no contribution to the energy density (2.20) from any finite $t$ ).

To show that, we shall rely on the expression for the emission time that can be read off eq. (2.14), that is,

$$
\dot{t}=\gamma^{2}(t-v x)-\gamma \sqrt{\boldsymbol{x}_{\perp}^{2}+z^{2}+\gamma^{2}(x-v t)^{2}} .
$$

Let us focus on a bit of string at a fixed value of $z$. We have just seen that, this particular piece of string will contribute to the backreaction at $x_{\perp}, \gamma(x-v t) \sim z$. When $\gamma \rightarrow \infty$, the response is peaked at $x-t \sim z / \gamma \rightarrow 0$ (as also manifest on eq. (2.20)) and then it is convenient to write $x \equiv t-\delta \bar{x} / \gamma$, where the quantity $\delta \bar{x} \sim z$ thus defined remains fixed as

\footnotetext{
${ }^{6}$ Note that for the uniformly moving heavy quark, the $4 \mathrm{D}$ velocity of the heavy quark on the boundary coincides with the $5 \mathrm{D}$ velocity of any bit of the vertical string in $\mathrm{AdS}_{5}$.
} 
$v \rightarrow 1$. Simple algebra using $1-v \simeq 1 / 2 \gamma^{2}$ as $v \rightarrow 1$ implies

$$
\begin{aligned}
& t-v x=t(1-v)+v \frac{\delta \bar{x}}{\gamma} \simeq \frac{t}{2 \gamma^{2}}+\frac{\delta \bar{x}}{\gamma}, \\
& x-v t=t(1-v)-\frac{\delta \bar{x}}{\gamma} \simeq \frac{t}{2 \gamma^{2}}-\frac{\delta \bar{x}}{\gamma},
\end{aligned}
$$

and hence

$$
\dot{t} \simeq \frac{t}{2}+\gamma \delta \bar{x}-\gamma \sqrt{\boldsymbol{x}_{\perp}^{2}+z^{2}+[(t / 2 \gamma)+\delta \bar{x}]^{2}} \rightarrow \gamma \delta \bar{x}-\gamma \sqrt{\boldsymbol{x}_{\perp}^{2}+z^{2}+\delta \bar{x}^{2}},
$$

where the last limit hold as $v \rightarrow 1$ at fixed $t$. Clearly $t<0$ for any $t$ and finite value of $\gamma$, and $\dot{t} \rightarrow-\infty$ when $\gamma \rightarrow \infty$, as anticipated.

For the problem at hand, where the $5 \mathrm{D}$ velocity of any bit of string is parallel to the Minkowski boundary, the above property is easy to understand: this is merely a consequence of Lorentz time dilation. But as we shall later discover, the same mathematical property - the fact that there is no boundary backreaction from sources which propagate inside $\mathrm{AdS}_{5}$ at the $5 \mathrm{D}$ speed of light - holds whatever the direction of motion of the sources inside the bulk and in particular for sources falling along $z$.

\subsection{Small pulse down the string}

Whereas the previous calculation was merely a warm-up exercice, which permitted us to fix the notations and introduce some general formulæ, the calculation to follow will provide us with the first non-trivial example of the phenomenon that we would like to emphasize throughout this paper: a source which radiates in the vacuum of the strongly coupled $\mathcal{N}=4$ SYM theory produces an energy density which exhibits no quantum broadening in the supergravity approximation.

Here, the source will be the heavy quark which is accelerated under the action of a weak $^{7}$ but otherwise arbitrary external force, and thus can radiate quanta of $\mathcal{N}=4$ SYM. From the perspective of the dual, gravity, calculation, this means that the endpoint of the string at the boundary is forced to follow some arbitrary motion, whose amplitude is weak and slowly varying in time (see below for the precise conditions). Via the (linearized) equations of motion, this perturbation of the endpoint propagates as a small perturbation of the shape of the string inside the bulk.

Furthermore, the lack of quantum broadening means that the energy density radiated by the quark - i.e., the backreaction produced by the string perturbation on the boundary - exhibits the same, localized, distribution in space and time as the corresponding solution of the classical Maxwell equations: the radiated energy appears to propagate at the 4D speed of light, so like classical fields or free massless quanta. As we shall see, the absence of broadening is a consequence of the fact that the string backreaction responsible for radiation is restricted to the string endpoint at $z=0$ alone.

For simplicity, we shall assume that the string is at rest in the absence of the external perturbation and that its deformation from a vertical line is restricted to just one spatial

\footnotetext{
${ }^{7}$ The case of a large, but uniform, angular acceleration has been studied in ref. [23] and will be also discusses in section 2.3 below; other cases will be considered in a subsequent paper [27].
} 
direction: the $x \equiv x^{1}$ axis. (If the latter condition is satisfied by the string endpoint, then it is automatically satisfied by the string perturbation anywhere in the bulk.) We shall present explicit results for arbitrary small perturbations, but the physically most transparent example is that of an external force which is localized in time - a 'kick' acting on the heavy quark. The associated string perturbation is a pulse which propagates down the string. The corresponding backreaction has been already computed in ref. [28], but only insofar as the 'dilaton' field (the expectation value of the operator $\operatorname{Tr} F_{\mu \nu}^{2}$ ) is concerned. As we shall later explain, that particular case is rather peculiar and in any case very different from the backreaction for the energy density.

Consider therefore a string which in the absence of any external perturbation is 'sitting' at $\boldsymbol{r}=0$. (The corresponding formulæ are obtained by letting $v \rightarrow 0$ in all the formulæ in section 2.1.) Under the action of an external force, the string endpoint acquires a timedependent deviation $x_{q}(t)$ along the $x$ axis, which is 'small' in a sense to be shortly specified. Then the general perturbation of the string can be parametrized as $x_{s}(t, z)$ with boundary condition $x_{s}(t, z=0)=x_{q}(t)$. The string embedding functions are $X^{M}=\left(t, x_{s}, 0,0, z\right)$ and the Nambu-Goto action takes the form

$$
S=-T_{0} \int \mathrm{d} t \mathrm{~d} z \sqrt{-g}=-T_{0} \int \mathrm{d} t \mathrm{~d} z\left|G_{00}\right| \sqrt{1-\dot{x}_{s}^{2}+x_{s}^{\prime 2}}
$$

with $G_{00}=-L^{2} / z^{2}$. Varying $x_{s} \rightarrow x_{s}+\delta x(t, z)$ and requiring the action to be stationary we obtain the classical string equation of motion (EOM)

$$
\frac{\partial}{\partial t} \frac{\dot{x}_{s}}{\sqrt{1-\dot{x}_{s}^{2}+x_{s}^{\prime 2}}}-\frac{1}{\left|G_{00}\right|} \frac{\partial}{\partial z} \frac{\left|G_{00}\right| x_{s}^{\prime}}{\sqrt{1-\dot{x}_{s}^{2}+x_{s}^{\prime 2}}}=0
$$

Assuming that $\left|\dot{x}_{s}\right|,\left|x_{s}^{\prime}\right| \ll 1$ the EOM linearizes in $x_{s}$ and becomes

$$
\left(\partial_{t}^{2}-\partial_{z}^{2}+\frac{2}{z} \partial_{z}\right) x_{s}=0
$$

The general retarded solution to eq. (2.28) with the given boundary condition is

$$
x_{s}(t, z)=x_{q}(t-z)+z \dot{x}_{q}(t-z) .
$$

Notice that this is the linear approximation to the general solution of the EOM [7]

$$
t=t_{q}+\gamma_{q} z, \quad x_{s}=x_{q}+\gamma_{q} \dot{x}_{q} z,
$$

where $x_{q}, \dot{x}_{q}$ and $\gamma_{q}$ are evaluated at $t_{q}$. Interestingly, the linear approximation is tantamount to letting $\gamma_{q} \rightarrow 1$, that is, it is tantamount to the non-relativistic approximation for the motion of the quark on the boundary. In general, it is helpful to think that the boundary motion can be parametrized as

$$
x_{q}=x_{0} f(t / \tau, \ldots),
$$

where $\tau$ is the smallest time scale in the problem and the dots stand for the dependence on the remaining time scales. Then one can see that the linear approximation holds under 
the conditions that $x_{0} \ll \tau$ and $z \ll \tau^{2} / x_{0}$. Thus, even for boundary perturbations whose amplitude $x_{0}$ is arbitrarily small, the linear approximation can be trusted only up to some maximal penetration $z$ inside the bulk, beyond which the string perturbation (which is amplified by the scale factor $z$ ) becomes relatively large.

To be more specific, let us consider a perturbation which is localized in time:

$$
x_{q}=x_{0} \exp \left(-\frac{t^{2}}{2 \sigma^{2}}\right) .
$$

In the linear approximation, this leads to the following string profile

$$
x_{s}=x_{0}\left[1+\frac{z(z-t)}{\sigma^{2}}\right] \exp \left[-\frac{(z-t)^{2}}{2 \sigma^{2}}\right] \text {, }
$$

which describes a pulse on the string with a maximum at $z=t$ and a width of order $\sigma$. Hence, the position of the peak propagates down the string at the $5 \mathrm{D}$ speed of light: $v_{z}=\mathrm{d} z / \mathrm{d} t=1$. The linear approximation is valid as long as $x_{0} \ll \sigma$ and $z \ll \sigma^{2} / x_{0}$; these conditions allow for values of $z$ which are much larger than $\sigma$.

Returning to the general perturbation (2.29), let us compute the radiated energy density, as produced via backreaction on the boundary. To that aim, we shall rely on the general formulæ introduced in section 2.1 and in particular on eq. (2.9) for the string stress tensor, where now $\delta^{(3)}\left(\boldsymbol{r}-\boldsymbol{r}_{s}\right)=\delta^{(2)}\left(\boldsymbol{x}_{\perp}\right) \delta\left(x-x_{s}\right)$. In what follows, we shall simplify these formulæ in order to (i) be consistent with the linear approximation for the string perturbation, and (ii) retain only those contributions which correspond to radiation.

The second constraint above turns out to be quite subtle: the string worldsheet is simultaneously describing various forms of energy - the Coulomb energy of the heavy quark, the radiated energy, and interference terms between the two - and in general it does not seem possible to unambiguously isolate these various contributions already at the level of the string stress tensor. Rather, the general strategy in that sense is to first compute the total energy density on the boundary (via backreaction from the string in the bulk) and then identify the radiation as the component of the energy density which shows the slowest fall-off, namely like $1 / r^{2}$, when $r \rightarrow \infty$. In our present analysis, we shall partly follow this strategy — by chasing contributions with a slow fall-off at large $r$ - but we shall combine it with a physical analysis of the string stress tensor $t_{M N}$, which will allow us to identify and discard the contributions to the Coulomb energy.

For more clarity, we relegate most of the technical details to appendix $\mathrm{C}$ and focus here on the main results and on the points of physics. A first conclusion of the analysis in appendix $\mathrm{C}$ is that, to the accuracy of interest, one can ignore the small deviation $x_{s}$ away from $x=0$ within the $\delta$-function expressing the support of the string; that is, one can replace $\delta^{(3)}\left(\boldsymbol{r}-\boldsymbol{r}_{s}\right) \rightarrow \delta^{(3)}(\boldsymbol{r})$. Indeed, the contributions obtained by expanding $\delta\left(x-x_{s}\right)$ in powers of $x_{s}$ represent, at most, interference effects between Coulomb energy and radiation and thus decay as $1 / r^{3}$ or faster at large distances. ${ }^{8}$ We therefore write

$$
t_{M N}=\tilde{t}_{M N} \delta^{(3)}(\boldsymbol{r}) .
$$

\footnotetext{
${ }^{8}$ Note that such an interference term represents the leading order correction to the 'dilaton' field due to the static string [28]. So, in that case, it was important to keep trace of $x_{s}$ inside $\delta\left(x-x_{s}\right)$.
} 
The various components $\tilde{t}_{M N}$ are listed in eq. (C.8) in appendix C. Here, we focus on the energy density $\tilde{t}_{M N}$, which reads:

$$
\tilde{t}_{00}=\frac{\sqrt{\lambda}}{2 \pi} \frac{z}{L^{3}} \frac{1+x_{s}^{\prime 2}}{\sqrt{1-\dot{x}_{s}^{2}+x_{s}^{\prime 2}}}, \quad E_{s}=\int_{z_{m}}^{\infty} \mathrm{d} z \frac{L^{3}}{z^{3}} \tilde{t}_{00}(t, z) .
$$

Given $\tilde{t}_{00}$, the total energy $E_{s}$ stored in the string is computed as shown above. As anticipated, $\tilde{t}_{00}$ also encodes the Coulomb energy, which would be non-vanishing even in the absence of acceleration. For instance, for a heavy quark with constant velocity $v^{1}=v$ (the problem discussed in section 2.1), we have $\dot{x}_{s}=v$ and $x_{s}^{\prime}=0$, and the above equations yield $E_{s}=m_{q} \gamma$, as expected for a relativistic particle. (Recall that $z_{m}=\sqrt{\lambda} /\left(2 \pi m_{q}\right)$ with $m_{q}$ the mass of the quark.) For a generic motion of the heavy quark, eq. (2.35) simultaneously describes Coulomb energy (associated with the instantaneous value of $\dot{x}_{s}$ ) and radiation (associated with the variation of $\dot{x}_{s}$, i.e. with the quark acceleration, and related to this - to the deformation of the string worldsheet, as measured by $x_{s}^{\prime}$ ). As we now demonstrate, these two types of energy can be disentangled from each other for the small perturbation problem at hand.

For consistency with the linear approximation in (2.29), we need to expand eq. (2.35) to quadratic order in the perturbation $x_{s}$. This yields

$$
\tilde{t}_{00} \simeq \frac{\sqrt{\lambda}}{2 \pi} \frac{z}{L^{3}}\left(1+\frac{1}{2} x_{s}^{\prime 2}+\frac{1}{2} \dot{x}_{s}^{2}\right) .
$$

At this level, it is convenient to use eq. (2.29) in order to relate $\dot{x}_{s}$ and $x_{s}^{\prime}$ to the boundary motion:

$$
\dot{x}_{s}=\dot{x}_{q}+z \ddot{x}_{q}, \quad x_{s}^{\prime}=-z \ddot{x}_{q},
$$

where in the r.h.s. $x_{q}$ is evaluated at $t-z$. By inserting these expressions in eq. (2.36) and using the fact that $\mathrm{d} / \mathrm{d} t=-\mathrm{d} / \mathrm{d} z$ when acting on $x_{q}$, we deduce

$$
\begin{aligned}
\tilde{t}_{00} & \simeq \frac{\sqrt{\lambda}}{2 \pi} \frac{z}{L^{3}}\left[1+\frac{1}{2}\left(\dot{x}_{q}^{2}+2 z \dot{x}_{q} \ddot{x}_{q}\right)+z^{2} \ddot{x}_{q}^{2}\right] \\
& =\frac{\sqrt{\lambda}}{2 \pi} \frac{z^{3}}{L^{3}}\left[\frac{1}{z^{2}}-\frac{1}{2} \frac{\mathrm{d}}{\mathrm{d} z}\left(\frac{\dot{x}_{q}^{2}}{z}\right)+\ddot{x}_{q}^{2}\right] .
\end{aligned}
$$

We shall now argue that the first two terms within the last square bracket, that is $1 / z^{2}$ and the term which is a total derivative, represent the Coulomb energy of the non-relativistic quark, whereas the last term, proportional to the quark acceleration squared, represents the radiation (see also refs. [7, 22] for similar arguments). To that aim, we compute the total string energy according to eq. (2.35):

$$
\begin{aligned}
E_{s} & \simeq \frac{\sqrt{\lambda}}{2 \pi} \int_{z_{m}}^{\infty} \mathrm{d} z\left[\frac{1}{z^{2}}-\frac{1}{2} \frac{\mathrm{d}}{\mathrm{d} z}\left(\frac{\dot{x}_{q}^{2}}{z}\right)+\ddot{x}_{q}^{2}\right] \\
& =\frac{\sqrt{\lambda}}{2 \pi z_{m}}\left(1+\frac{v^{2}}{2}\right)+\frac{\sqrt{\lambda}}{2 \pi} \int_{z_{m}}^{\infty} \mathrm{d} z \ddot{x}_{q}^{2}(t-z) \\
& =m_{q}+\frac{m_{q} v^{2}}{2}+\frac{\sqrt{\lambda}}{2 \pi} \int_{-\infty}^{t} \mathrm{~d} \dot{t} \ddot{x}_{q}^{2}(\dot{t}),
\end{aligned}
$$


where $v=\dot{x}_{q}(t)$. As anticipated, the first two terms in the last expression are recognized as the quark energy in the non-relativistic approximation, whereas the remaining integral involving $\ddot{x}_{q}^{2}$ is clearly the radiated energy.

Incidentally, the above calculation already tells us what should be the total energy radiated by the quark: by energy conservation, this is the same as the radiative piece of the energy stored into the string. Hence,

$$
E_{\mathrm{rad}}=\frac{\sqrt{\lambda}}{2 \pi} \int_{-\infty}^{t} \mathrm{~d} \dot{t} \ddot{x}_{q}^{2}(\dot{t}), \quad P \equiv \frac{\mathrm{d} E_{\mathrm{rad}}}{\mathrm{d} t}=\frac{\sqrt{\lambda}}{2 \pi} a^{2},
$$

where $a=\ddot{x}_{q}$ is the quark acceleration and $P$ is the radiated power. These formulæ were to be expected: as shown by Mikhailov [7], they hold for an arbitrary motion of the quark on the boundary. Moreover, they are formally identical with the corresponding classical result in electrodynamics, up to the replacement $\sqrt{\lambda} \leftrightarrow e^{2} / 3$. Recovering these results via the explicit calculation of the string backreaction represents a non-trivial check on the respective calculation, to which we now return.

Via similar manipulations, one can convince oneself that the radiative contributions to all the components of $\tilde{t}_{M N}$ are proportional to $a^{2}$ (to the accuracy of interest). The respective results are quite simple: the only non-trivial components are

$$
\tilde{t}_{00}^{\mathrm{rad}}=\tilde{t}_{55}^{\mathrm{rad}}=-\tilde{t}_{05}^{\mathrm{rad}}=\frac{\sqrt{\lambda}}{2 \pi} \frac{z^{3}}{L^{3}} \ddot{x}_{q}^{2}
$$

with $\ddot{x}_{q}^{2}$ evaluated at $t-z$. Using these results, one can evaluate the radiated energy density according to eqs. (2.5) and (2.6). It turns out that $\mathcal{E}_{\mathrm{B}}=0$ whereas

$$
\mathcal{E}_{\mathrm{A}}=\frac{\sqrt{\lambda}}{\pi^{2}} \partial_{r^{2}}^{2} \int \mathrm{d} z \mathrm{~d} \dot{t} \delta(\mathcal{W}) z(z+t-\dot{t}) \ddot{x}_{q}^{2}(\dot{t}-z) .
$$

The linear approximation is a priori restricted to relatively small values of $z$, but this poses no problem for the above integral over $z$, since this is actually saturated by its lower limit at $z_{m} \approx 0$ (see below). Also, $\mathcal{W}$ has to be evaluated at $x_{s}=0$, cf. eq. (2.34), and therefore (for the retarded solution)

$$
\delta(\mathcal{W})=\frac{\delta\left(\dot{t}-t+\sqrt{z^{2}+r^{2}}\right)}{2 \sqrt{z^{2}+r^{2}}} .
$$

By using this $\delta$-function to perform the time integration, we obtain

$$
\mathcal{E}=\frac{\sqrt{\lambda}}{2 \pi^{2}} \partial_{r^{2}}^{2} \int \mathrm{d} z \frac{z\left(z+\sqrt{z^{2}+r^{2}}\right)}{\sqrt{z^{2}+r^{2}}} \ddot{x}_{q}^{2} .
$$

with $\ddot{x}_{q}^{2}$ evaluated at $\dot{t}-z=t-z-\sqrt{z^{2}+r^{2}}$. At this point, it is convenient to change the integration variable from $z$ to $\xi$, via

$$
\xi \equiv z+\sqrt{z^{2}+r^{2}}
$$

This implies

$$
z=\frac{\xi^{2}-r^{2}}{2 \xi}, \quad \mathrm{d} z=\frac{\xi^{2}+r^{2}}{2 \xi^{2}} \mathrm{~d} \xi, \quad \sqrt{z^{2}+r^{2}}=\frac{\xi^{2}+r^{2}}{2 \xi},
$$


and the energy density becomes (at the lower limit, we neglect $z_{m}$ next to $r$ )

$$
\mathcal{E}=\mathcal{E}_{\mathrm{A}}=\frac{\sqrt{\lambda}}{4 \pi^{2}} \partial_{r^{2}}^{2} \int_{r}^{\infty} \frac{\mathrm{d} \xi}{\xi}\left(\xi^{2}-r^{2}\right) \ddot{x}_{q}^{2},
$$

where now the argument of $x_{q}$ is $t-\xi$ and every dot corresponds to a derivative w.r.t. $t$ or equivalently the argument.

At this point, an essential simplification occurs: since the integrand in eq. (2.47) is linear in $r^{2}$, it is quite clear that, after we take the external derivatives, the only surviving contribution is a boundary term, coming from the lower boundary at $\xi=r$, or $z=0$. A similar property was noticed in ref. [23] in relation with the rotating string. On the other hand, the situation is very different for the calculation of the average 'dilaton' density $\left\langle\operatorname{Tr} F_{\mu \nu}^{2}\right\rangle$ on the boundary, where a final integration over all values of $\xi$ is left to be done [28]. This difference has dramatic consequences for the space-time pattern of the response on the boundary, which we shall shortly discuss.

Returning to eq. (2.47), it is important to stress that the simplification alluded to above has occurred because of compensations between the various terms in eq. (2.5), which in turn were made possible by the particularly simple structure of the radiation piece of the bulk stress tensor, eq. (2.41). (For instance, if one takes just the $\tilde{t}_{00}$ piece in the integrand of eq. (2.5) and then uses the simple expression for $\tilde{t}_{00}$ shown in eq. (2.41), one obtains terms $\propto r^{4}$ which would yield non-trivial contributions from the bulk.) We conclude that both the particular tensor structure in eq. (2.41), and the specific functional form of the individual components there, are important for our final conclusion that only boundary terms survive. These boundary terms are easily evaluated as

$$
\mathcal{E}(t, r)=\frac{\sqrt{\lambda}}{8 \pi^{2}} \frac{\ddot{x}_{q}^{2}(t-r)}{r^{2}} .
$$

The total radiated energy is obtained by multiplying the above expression with $4 \pi r^{2}$ and integrating over $r$. Clearly, this yields the expected result, cf. eq. (2.40).

From the previous discussion, it should be clear that eq. (2.48) represents the general solution for an arbitrary one-dimensional motion of the heavy quark in the non-relativistic limit. In particular, for the Gaussian pulse (2.33) one finds

$$
\mathcal{E}=\frac{\sqrt{\lambda}}{8 \pi^{2}} \frac{x_{0}^{2}}{\sigma^{8} r^{2}}\left[(r-t)^{2}-\sigma^{2}\right]^{2} \exp \left[-\frac{(r-t)^{2}}{\sigma^{2}}\right], \quad E_{\mathrm{rad}}=\frac{3 \sqrt{\lambda}}{8 \sqrt{\pi}} \frac{x_{0}^{2}}{\sigma^{3}}
$$

The most important feature of eq. (2.48) for us here is the fact that the space-time distribution of the radiated energy is controlled by the function $x_{q}(t-r)$, i.e. by the initial perturbation of the heavy quark translated from $r=0$ (the quark position) to $r=t$. A similar pattern would be obtained by solving the classical Maxwell equations with the heavy quark as a source. It looks like, at strong coupling, the radiation is simply propagating from $r=0$ up to $r=t$ at the speed of light, in the same way as classical radiation (or free massless quanta) would do. In particular, for the localized perturbation (2.32), the radiated energy density, eq. (2.49), propagates as a spherical shell centered at $r=t$ and with a width equal to the width $\sigma$ of the initial perturbation. There is no sign of 
quantum broadening, that is, no time-like components in the radiation left behind at $r \ll t$, in contrast to expectations based on the physical picture of parton branching at strong coupling (see the discussion at the beginning of section 3 ).

In what follows, we shall argue that this lack of broadening of the radiative energy density on the boundary is a consequence of the fact that the whole backreaction comes from the string endpoint at $z=0$, as noticed after eq. (2.47). To that aim, we shall show that the backreaction from string points at $z>0$ - if non-zero! - would introduce a spread $t-r \sim z$ in the energy distribution, which with increasing $z$ could become arbitrarily large. For more clarity we shall focus on a localized initial perturbation, like eq. (2.32). Then the perturbation propagates down the string as a pulse centered at $z=t$ (cf. eq. (2.33)). This property together with the causality condition $\mathcal{W}=0$ implies the following relation between the position $z$ of the 'source in the bulk' (here, a point on the pulse) and the measurement point $(t, r)$ on the boundary: $t \simeq z+\sqrt{z^{2}+r^{2}}$ or

$$
z \simeq \frac{t^{2}-r^{2}}{2 t} \simeq \frac{t-r}{2}
$$

where the second approximation holds when both $t$ and $r$ are large with $t, r \gg t-r$. This condition is easy to understand: it takes a time $t \simeq z$ for the perturbation to propagate along the string from $z=0$ up to $z$ and then a time $\sqrt{z^{2}+r^{2}}$ for the gravitational wave expressing the backreaction to propagate through $\mathrm{AdS}_{5}$ from the point $z$ on the string (which has $r=0$ ) up to the point $r$ on the boundary. Note that both propagations alluded to above proceed at the $5 \mathrm{D}$ speed of light.

Eq. (2.50) is the expected form of the UV/IR correspondence - or, more precisely, its expression (ii) according to the discussion in the Introduction. It predicts a spreading $t-r$ in the energy distribution which grows proportionally with the radial location $z$ of the source in the bulk. Such a spreading is seen indeed in the calculation of the 'dilaton' density on the boundary [28], but it is absent from the corresponding calculations of the radiation, as presented here and in ref. [23]. Clearly, this absence of broadening is still consistent with eq. (2.50) because in the case of radiation the whole backreaction comes from the endpoint of the convolution at $z=z_{m}$. Yet, this is a rather surprising feature, that we would like to understand better via the remaining analysis in this paper.

\subsection{Velocity of a bit of energy}

In this subsection, we start developing an argument, to be completed at later stages of this analysis, which sheds more light on the absence of broadening in the SUGRA calculations of radiation. Namely, we shall argue that the lack of backreaction from bulk sources which lie far away from the boundary is a consequence of the fact that these sources propagate at the speed of light in $\mathrm{AdS}_{5}$. We should stress from the very beginning that this property is not just a consequence of the kinematics, and hence of causality. Indeed, we have just seen, towards the end of the previous subsection, that causality alone does permit backreaction (in terms of radiated energy on the boundary) from bulk points at $z>0$ and that the respective contributions would show spreading, cf. eq. (2.50). The fact that such contributions are nevertheless absent in the final result is therefore a property of the 
SUGRA dynamics, that we shall now attribute to the propagation of bulk sources at the $5 \mathrm{D}$ speed of light.

The argument will be developed in several steps: First, in the present subsection, we shall verify that the flow of energy in $\mathrm{AdS}_{5}$ proceeds indeed at the speed of light (except near $z=0$ ) for the two examples that we have discussed so far: the small string perturbation and the rotating string of ref. [23]. Then, in section 3.1 we shall show that this property - the propagation of bulk sources at the speed of light - implies the particular structure for the bulk stress tensor shown in eq. (2.41), which in turn is responsible for the lack of backreaction (as discussed after eq. (2.47)). Finally in section 4.1 we shall argue that the fact bulk sources propagating at the speed of light cannot radiate may be interpreted as a form of Lorentz time dilation, but in a 'twisted' system of coordinates which mixes the 5 th dimension with one of the spatial coordinates $x^{i}$ on the boundary.

In the context of section 2.2 , the fact that the perturbation propagates down the string at the speed of light seems already well established: we have noticed e.g. that the peak of the pulse in eq. (2.33) travels according to $z=t$. However, other examples like the rotating string [23] or the trailing string (at finite temperature) $[8,9]$ show that it is not always possible to associate the flow of energy along a string with a propagating string deformation. So, we need a more precise definition of what we mean by the 'velocity of the energy flow down the string'. This is provided by the string stress tensor $t^{M N}$ : the component $t^{0 M}$ (with $M \neq 0$ ) describes the energy flow in the $M$ direction, while $t^{00}$ is the energy density; hence the ratio $v_{M}=t^{0 M} / t^{00}$ defines the respective component of the velocity. ${ }^{9}$ As explained in section 2.2, care must be taken to include into $t^{0 M}$ and $t^{00}$ only the respective radiative contributions, since the Coulomb energy is not flowing. We shall therefore use

$$
v_{M}=\frac{t_{\mathrm{rad}}^{0 M}}{t_{\mathrm{rad}}^{00}} .
$$

For instance, this definition together with eq. (2.41) immediately implies $v_{z}=1$ (and of course $v_{i}=0$ for $\left.i=1,2,3\right)$, at any $z>0$. So, for that problem, we have $v^{2}=v_{z}^{2}=1$, as anticipated.

An alternative definition, which is equivalent to eq. (2.51) but often easier to use in practice, involves the worldsheet stress tensor $\pi_{M}^{a}$ with $a=\tau, \sigma$. Within the string worldsheet, there is only one direction for the energy flow, namely $\sigma=z$. (As before, $\tau=t$ and $\sigma=z$.) Then it is natural to define the radial velocity of a bit of energy as

$$
\left.v_{z} \equiv \frac{\mathrm{d} E_{s} / \mathrm{d} t}{\mathrm{~d} E_{s} / \mathrm{d} z}\right|_{\mathrm{rad}},
$$

where $\mathrm{d} E_{s} / \mathrm{d} z$ and $\mathrm{d} E_{s} / \mathrm{d} t$ denote the energy density and the energy flow down the string, respectively, and the subscript "rad" indicates that we have to consider only radiative

\footnotetext{
${ }^{9}$ Note that $v_{M}$ is not a $5 \mathrm{D}$ vector, so the position of the index $M$ is irrelevant.
} 
contributions to these quantities. One generally has (with $T_{0}=\sqrt{\lambda} / 2 \pi L^{2}$ )

$$
\begin{aligned}
& \frac{\mathrm{d} E_{s}}{\mathrm{~d} z}=-\pi_{0}^{\tau}=\frac{T_{0}}{\sqrt{-g}}\left[\left(\dot{X} \cdot X^{\prime}\right) X_{0}^{\prime}-\left(X^{\prime} \cdot X^{\prime}\right) \dot{X}_{0}\right], \\
& \frac{\mathrm{d} E_{s}}{\mathrm{~d} t}=-\pi_{0}^{\sigma}=\frac{T_{0}}{\sqrt{-g}}\left[\left(\dot{X} \cdot X^{\prime}\right) \dot{X}_{0}-(\dot{X} \cdot \dot{X}) X_{0}^{\prime}\right] .
\end{aligned}
$$

One way to understand eq. (2.52) is to follow a curve of constant energy $E_{s}(t, z)$. The condition $\mathrm{d} E_{s}=0$ under small variations $\mathrm{d} t$ and $\mathrm{d} z$ implies indeed

$$
\mathrm{d} E_{s}=\frac{\partial E_{s}}{\partial t} \mathrm{~d} t+\frac{\partial E_{s}}{\partial z} \mathrm{~d} z=0 \Longrightarrow v_{z} \equiv \frac{\mathrm{d} z}{\mathrm{~d} t}=\frac{-\partial E_{s} / \partial t}{\partial E_{s} / \partial z} .
$$

The difference in sign w.r.t. eq. (2.52) comes from the fact that, in eq. (2.53), we have defined the energy flux to be positive when the energy is decreasing at a given $z$.

It is straightforward to apply eqs. (2.52)-(2.53) to the problem of a small string perturbation in section 2.2 and thus rederive the expected result $v_{z}=1$. (The separation of the radiated energy within the worldsheet tensor proceeds in exactly the same way as for the respective 5D stress tensor, cf. the discussion leading to eq. (2.41).) Here we shall rather use them for the problem of the rotating string, i.e. a string attached to a heavy quark which rotates on the boundary at uniform angular velocity $\omega_{0}$. The corresponding backreaction has been computed in ref. [23] and found to arise from the string endpoint at $z=0$ alone. In agreement with that finding, we shall now show that the velocity of a bit of energy flowing down the string is equal to one at any $z>0$. In spherical coordinates, the profile of the rotating string reads [23]

$$
r_{s}=\sqrt{R_{0}^{2}+\gamma_{0}^{2} v_{0}^{2} z^{2}}, \quad \theta_{s}=\frac{\pi}{2}, \quad \phi_{s}=\omega_{0}\left(t-\gamma_{0} z\right)+\arctan \left(\gamma_{0} \omega_{0} z\right),
$$

where $\omega_{0}=v_{0} / R_{0}, \gamma_{0}^{2}=1 /\left(1-v_{0}^{2}\right)$ and with $v_{0}$ the magnitude of the boundary velocity. Inserting these formulæ into eq. (2.53), it is straightforward to deduce that

$$
\begin{aligned}
\frac{\mathrm{d} E_{s}}{\mathrm{~d} z} & =\frac{\sqrt{\lambda}}{2 \pi} \frac{\gamma_{0}}{z^{2}}+\frac{\sqrt{\lambda}}{2 \pi} \gamma_{0} a_{0}^{2}, \\
\frac{\mathrm{d} E_{s}}{\mathrm{~d} t} & =\frac{\sqrt{\lambda}}{2 \pi} a_{0}^{2} .
\end{aligned}
$$

Here $a_{0}=\gamma_{0}^{2} v_{0}^{2} / R_{0}$ is the proper acceleration of the boundary motion, which also determines the position of the induced worldsheet horizon: $z_{\mathrm{h}}=1 / a_{0}$. Considering the energy density in eq. (2.56), it seems natural to associate the first term with the Coulomb energy density $\mathrm{d} E_{\text {Coul }} / \mathrm{d} z$ of the rotating quark, and the second one with the energy density due to radiation, $\mathrm{d} E_{\mathrm{rad}} / \mathrm{d} z$. This identification is supported by the type of arguments leading to eq. (2.41): after integrating eq. (2.56) over $z$ to compute the total energy stored in the string, one finds that the first term there yields a contribution $m_{q} \gamma_{0}$, which is recognized as the energy of a relativistic quark with uniform velocity $v_{0}$. As for the energy flux in eq. (2.57), this coincides with the expected result for radiated power, so it is clear that this includes radiation alone. Hence, the radial velocity of a bit of energy is given by

$$
v_{z}=\frac{\mathrm{d} E_{s} / \mathrm{d} t}{\mathrm{~d} E_{\mathrm{rad}} / \mathrm{d} z}=\frac{1}{\gamma_{0}} .
$$


This is strongly suppressed in the ultrarelativistic limit $\gamma_{0} \gg 1$, which can be interpreted as a consequence of the Lorentz collimation of the radiated energy around the direction of emission (here, in the five-dimensional sense). But a small progression in $z$ along the profile of the string implies a relatively large displacement in the spatial direction $r$, since the respective coordinate $r_{s}$ of the string rises rapidly with $z$, cf. eq. (2.55). Specifically, the energy bit has a non-zero velocity $v_{r}$, computed as

$$
v_{r} \equiv \frac{\mathrm{d} r_{s}}{\mathrm{~d} t}=\dot{r}_{s}+r_{s}^{\prime} v_{z}=\frac{\gamma_{0} v_{0}^{2} z}{\sqrt{R_{0}^{2}+\gamma_{0}^{2} v_{0}^{2} z^{2}}},
$$

with of course $\dot{r}_{s}=0$ for the rotating string. Finally, the string as a whole is rotating, and therefore so does the energy bit flowing along it. This implies the following $v_{\phi}$ component for the velocity of the energy flow

$$
v_{\phi} \equiv r_{s} \frac{\mathrm{d} \phi_{s}}{\mathrm{~d} t}=r_{s} \dot{\phi}_{s}+r_{s} \phi_{s}^{\prime} v_{z}=\frac{v_{0} R_{0}}{\sqrt{R_{0}^{2}+\gamma_{0}^{2} v_{0}^{2} z^{2}}} .
$$

(The other angular velocity vanishes, $v_{\theta}=0$, since $\theta$ is fixed for the string.) On the boundary $(z=0)$, one has $v_{\phi}=v_{0}$ with $v_{r}$ vanishing, while for large $z$, on the contrary, $v_{\phi}$ vanishes with $v_{r} \rightarrow v_{0}$. For the magnitude squared we obtain

$$
v^{2} \equiv v_{z}^{2}+v_{r}^{2}+v_{\phi}^{2}=1
$$

for any $z$ and for any boundary velocity $v_{0}$. As a check, notice that we would obtain the same results by computing the velocity of the energy flow directly from the 5D string stress tensor, according to eq. (2.51). The components of interest are the energy and momentum density given in spherical coordinates by [23]

$$
t_{\mathrm{rad}}^{0 M}=\frac{\sqrt{\lambda} \gamma_{0} z^{5}}{2 \pi L^{7}} \delta^{(3)}\left(\boldsymbol{r}-\boldsymbol{r}_{s}\right)\left(r_{s}^{\prime 2}+r_{s}^{2} \phi_{s}^{\prime 2},-\omega_{0} r_{s}^{2} r_{s}^{\prime} \phi_{s}^{\prime}, 0, \omega_{0} r_{s}^{\prime 2},-\omega_{0} r_{s}^{2} \phi_{s}^{\prime}\right)
$$

with the elements corresponding to $(t, r, \theta, \phi, z)$ respectively and where we have again subtracted from $t^{00}$ and $t^{0 \phi}$ the terms which correspond to the static energy of the string. (We shall elaborate more on this subtraction in the subsequent publication [27].) Then the velocity of the energy flow is

$$
v_{M}=\frac{1}{t_{\mathrm{rad}}^{00}}\left(t_{\mathrm{rad}}^{0 r}, t_{\mathrm{rad}}^{0 \theta}, r_{s} t_{\mathrm{rad}}^{0 \phi}, t_{\mathrm{rad}}^{0 z}\right),
$$

with $M=r, \theta, \phi, z$. Plugging eq. (2.62) into eq. (2.63) we arrive at the previously found expressions in eqs. (2.58), (2.59) and (2.60) for the components of the velocity.

Let us also comment here on some other intriguing result in ref. [23], namely the fact that the whole contribution to the energy density on the boundary, and not just its radiative part, appears to be generated by backreaction from the endpoint of the string at $z=0$. Whereas for radiation this feature can be ascribed to the propagation of the bits of energy down the string at the speed of light, there is certainly no such a correlation for the Coulomb piece of the energy, which is static. Rather, what we would like to argue here 
is that, for the case of the Coulomb energy, the respective finding is merely a consequence of a particular choice made in ref. [23] for the integration variables in the convolutions expressing the backreaction. Namely, the Coulomb energy appears as a bulk contribution (i.e., a result of the backreaction from string points at $z>0$ ) when using the standard variables $t$ and $z$ - in terms of which we have a transparent physical interpretation and the UV/IR correspondance holds as expected - , but it can be formally transferred into a boundary contribution (a backreaction from $z=0$ alone) via an appropriate change of variables mixing $t$ and $z$. To illustrate this point, we shall perform in appendix $\mathrm{A}$ the corresponding change of variables on the example of a string (heavy quark) with constant velocity and show that, with the new variables, the complete result appears to be generated at the endpoint of the integration at $z=0$. Yet, from the discussion in section 2.1, we already know that string points at any value of $z$ do contribute to the Coulomb energy, provided one uses the 'physical' variables $t$ and $z$. This example shows that care must be taken when trying to understand the UV/IR correspondence in various sets of coordinates.

At this point, one may wonder whether a similar property could also hold for the radiated energy - namely, whether the fact that the whole contribution to radiation is coming from $z=0$ is not just an artifact of our peculiar choice of coordinates. We do not believe this to be so, since in this case the correlation with $z=0$ has a clear signature, which is independent of the choice of integration variables in the calculation of backreaction: the lack of broadening of the radiated energy density in physical space. As we saw on the example of the small string perturbation, the width $t-r$ of the energy distribution on the boundary, eq. (2.49), is fixed by the width $\sigma$ of the initial perturbation. Via the UV/IR correspondence, this allows for contributions from string points at $z \lesssim 1 / \sigma$, but not from much larger values of $z$. So, whatever choice we make for the integration variables, the backreaction must come from points $z$ near the boundary, although it is likely that our previous respective choices were indeed special, in the sense that, with these choices, the whole contribution was generated from $z=0$ alone.

Let us conclude this section with a comment on the generality of our conclusions: the correlation between the bulk propagation at the speed of light and the lack of energy backreaction on the boundary implies that the absence of broadening for radiation should be a generic feature of the supergravity calculations. Indeed, whatever is the physical source of radiation in the dual gauge theory, the corresponding energy flow in $\mathrm{AdS}_{5}$ will propagate at the speed of light, except possibly for a transition region at small $z$, where the radiation is still connected to its source, and whose extent is controlled by the width of the external perturbation. Hence, the maximal broadening on the boundary will be fixed by that width as well. In the next section we shall give other examples of that type, where the bulk excitation is a supergravity field rather than a Nambu-Goto string.

\section{A time-like wave-packet}

In this section, we shall consider a different type of radiation, that emitted by a time-like (or 'massive') wave-packet, which decays into the massless quanta of $\mathcal{N}=4 \mathrm{SYM}$. The prototype of this phenomenon is the evolution of the time-like photon produced in $e^{+} e^{-}$ 
annihilation. To lowest order in perturbation theory at weak coupling, the photon decays into a pair of quanta (say, a quark and an antiquark) which in the center of mass frame propagate with equal but opposite momenta, at the speed of light. The final state in this approximation is simply a pair of back-to-back partons ('two jets'). If one includes higher order corrections (again, at weak coupling), then the pair of partons initially produced by the decay of the virtual photon are themselves off-shell (time-like) and can radiate other quanta. Then the final state consists in three or more partons. The stronger the coupling, the larger is the probability of branching in the final state, and the more complex is the structure of the latter in terms of partons. In the strong coupling limit, we expect this branching to be so efficient that the energy-momentum distribution in the final state consisting in myriads of partons - is essentially isotropic (in the center of mass of the virtual photon, of course). This expectation has been confirmed by the explicit AdS/CFT calculation of the angular correlations of the energy density in the final state, by Hofman and Maldacena [15], which revealed that there are no such correlations at all: the final state is fully isotropic, in a given event.

The physical picture of parton branching alluded to above has also another implication: it suggests that the energy-momentum distribution in the final state at strong coupling must be time-like. Namely, one expects the energy density to be non-zero essentially everywhere inside a three-dimensional sphere with radius $r=t$. (We assume that the initial photon wave-packet was localized near $r=0$.) Indeed, most of the quanta produced in the intermediate stages of the parton cascade are time-like and hence propagate with velocities smaller than one, thus yielding a tail in the energy distribution at $r<t$. This is to be contrasted with the energy distribution produced by a classical source localized at $t=0$ and $r=0$, which is a narrow spherical shell with $r=t$.

Yet, as we shall shortly see, the AdS/CFT calculation of the energy distribution produced by the time-like (TL) wave-packet at strong coupling (in the supergravity approximation) produces a narrow spherical shell which propagates outwards at the (4D) speed of light, so like a classical perturbation! The mathematical origin of this result is the same as for the string perturbation discussed in the previous section: the supergravity field describing the $\mathrm{AdS}_{5}$ perturbation induced by the TL wave-packet propagates into the bulk at the 5D speed of light and therefore has no backreaction in terms of energy density on the boundary. The latter is fully generated at early times, when the perturbation is close to the boundary (within a distance set by the width of the initial wave-packet) and its velocity is still smaller than one.

Whereas the mathematics of the backreaction calculation is quite similar to that of the string perturbation in section 2.2, the physical interpretation is perhaps sharper for the case of the TL wave-packet. This will allow us to better appreciate the inconsistency of the supergravity result with respect to general expectations from quantum mechanics.

In section 2.2 we have seen that the small pulse propagating down a vertical string behaves very much like a massless classical particle falling into $\mathrm{AdS}_{5}$ at the speed of light. In section 3.2 below, we shall discover that a similar picture holds also for the TL wavepacket. We therefore begin our discussion in this section by computing the backreaction due to a classical, massless, particle which undergoes free motion in $\mathrm{AdS}_{5}$. 


\subsection{The falling massless particle}

The motion of a free particle in a curved space-time follows a geodesic. For a massless particle in $\mathrm{AdS}_{5}$ and with our choice (2.1) for the metric, the most general such trajectory can be cast into the form

$$
x \equiv x^{1}=v t, \quad x^{2}=x^{3}=0, \quad z=\frac{1}{\gamma} t, \quad x^{2}+z^{2}=t^{2},
$$

where $\gamma=1 / \sqrt{1-v^{2}}$ is the boost factor associated with the longitudinal motion along the boundary. For the examples considered in sections 2.2 and 3.2 we actually need ${ }^{10}$ $v=0$, but for the present discussion it is not more difficult to consider a general value $v<1$. Note that eq. (3.1) together with the fact that $z \geq 0$ restricts the time variable to positive values, $t \geq 0$. That is, here we do not consider a steady situation, which would be translationally invariant in time, but a situation where the particle is put at $t=0$ on the Minkowski boundary and left to freely fall into $\mathrm{AdS}_{5}$.

To construct the associated bulk energy-momentum tensor, it is convenient to start with the expression valid for a massive particle with mass $m$, and then take the limit $m \rightarrow 0$. Then the $5 \mathrm{D}$ stress tensor reads

$$
t^{M N}=\frac{m}{\sqrt{-g}} \frac{\mathrm{d} x^{M}}{\mathrm{~d} t} \frac{\mathrm{d} x^{N}}{\mathrm{~d} t} \frac{\mathrm{d} t}{\mathrm{~d} \tau} \delta^{(4)}\left(x-x_{p}(t)\right),
$$

with $\tau$ the proper time and $x_{p}(t)$ the particle trajectory. We have

$$
\mathrm{d} \tau=\sqrt{-g_{00}} \frac{\mathrm{d} t}{\gamma}
$$

Furthermore, the momentum in local inertial coordinates can be expressed in terms of the conserved momentum as

$$
m \gamma=\frac{E_{0}}{\sqrt{-g_{00}}}
$$

so that eq. (3.2) becomes

$$
t^{M N}=\frac{E_{0}}{\left|g_{00}\right| \sqrt{-g}} \frac{\mathrm{d} x^{M}}{\mathrm{~d} t} \frac{\mathrm{d} x^{N}}{\mathrm{~d} t} \delta^{(4)}\left(x-x_{p}(t)\right) .
$$

At this level we can take the massless limit and specialize to $\mathrm{AdS}_{5}$ and the trajectory in eq. (3.1). One immediately finds (below, $\left.X^{M}=(t, v t, 0,0, t / \gamma)\right)$

$$
t^{M N}=E_{0}\left(\frac{z}{L}\right)^{7} \delta(x-v t) \delta^{(2)}\left(\boldsymbol{x}_{\perp}\right) \delta(z-t / \gamma) \frac{X^{M} X^{N}}{t^{2}} .
$$

After lowering the indices and letting $v=0$, this yields

$$
t_{00}=t_{55}=-t_{05}=E_{0}\left(\frac{z}{L}\right)^{3} \delta^{(3)}(\boldsymbol{r}) \delta(z-t), \quad t_{i M}=0,
$$

\footnotetext{
${ }^{10}$ Note that when comparing the present discussion to that in section 2.2 , the 'free particle' which is currently under consideration corresponds to the small pulse falling down the string, and not to the heavy quark that the string is attached to.
} 
which is very similar to the stress tensor (2.41) for the radiation emitted by a small string perturbation. The only, inessential, difference between eqs. (2.41) and (3.7) is that, in the latter, the bulk energy density is a strict $\delta$-function at $z=t$, while in the former it has some width around the maximum at $z=t$. Thus, the fact that the source in the bulk propagates at the speed of light along the radial direction $\left(v_{z}=1\right)$ automatically implies the tensor structure in (2.41) or (3.7) for the associated bulk stress tensor.

Using the general formulæ in section 2.1, it is straightforward to compute the boundary energy density produced via backreaction from the bulk stress tensor (3.6). One thus finds that $\mathcal{E}_{\mathrm{B}}=0$. Furthermore, within the integrand of eq. (2.5) we can write

$z\left(2 t_{00}-t_{55}\right)-(t-\hat{t}) t_{05}+(x-\dot{x})^{i} t_{i 5}=E_{0}\left(\frac{z}{L}\right)^{3} \frac{t+v x}{\gamma} \delta(\dot{x}-v \dot{t}) \delta^{(2)}\left(\dot{x}_{\perp}\right) \delta(z-\dot{t} / \gamma)$.

Using the $\delta$-functions above to perform the integrations over $\dot{x}, \dot{x}_{\perp}$ and $z$, one obtains

$$
\mathcal{E}=\mathcal{E}_{A}=\frac{2 E_{0}}{\pi} \frac{t+v x}{\gamma^{2}} \partial_{r^{2}}^{2} \int_{0}^{\infty} \mathrm{d} \hat{t} \hat{t} \delta\left(t^{2}-r^{2}-2(t-v x) \hat{t}\right)
$$

Note that the $\delta$-function inside the integrand together with the lower limit on $t$ (which was generated by the condition $z \geq 0$ ) imply a factor $\Theta\left(t^{2}-r^{2}\right)$. This is the expected condition, following from causality, that the space-time distribution of the energy density on the boundary must be time-like. However, this $\Theta$-function becomes a $\delta$-function after performing the two external derivatives w.r.t. $r^{2}$. One has indeed

$$
\mathcal{E}=\frac{E_{0}}{2 \pi \gamma^{2}} \frac{t+v x}{(t-v x)^{2}} \partial_{r^{2}}^{2}\left[\left(t^{2}-r^{2}\right) \Theta\left(t^{2}-r^{2}\right)\right]=\frac{E_{0}}{2 \pi \gamma^{2}} \frac{t+v x}{(t-v x)^{2}} \delta\left(t^{2}-r^{2}\right) .
$$

eq. (3.9) describes a spherical shell of zero width which propagates at the speed of light, so like classical radiation, and whose shape looks anisotropic because of the boost with velocity $v$ in the $x$ direction. By taking $v \rightarrow 0$, isotropy becomes manifest:

$$
\mathcal{E}=\frac{E_{0}}{4 \pi r^{2}} \delta(t-r), \quad \frac{\mathrm{d} E}{\mathrm{~d} \Omega} \equiv \int \mathrm{d} r r^{2} \mathcal{E}=\frac{E_{0}}{4 \pi} .
$$

In the above equation we have also shown the energy density per unit solid angle. Clearly, the total energy is equal to $E_{0}$ and coincides, as expected, with the total energy stored in the bulk stress tensor (3.7).

Returning to the calculation of the energy density according to eq. (3.8), we note that the condition $t=r$ implies $t=z=0$. That is, the whole backreaction on the boundary comes from the string endpoint at $z=0$, so like for the string perturbation in section 2.2. In both cases, this property follows from the fact that the source in the bulk - the pulse on the string, or the falling particle - propagates at the 5D speed of light.

To conclude this discussion, let us mention that for the bulk excitation under discussion - a free, point-like, massless particle with the stress tensor (3.6) - the backreaction on the $\mathrm{AdS}_{5}$ metric can be computed exactly, and not only in the linear approximation in which hold our general formulæ $(2.5)-(2.6)$ for $\mathcal{E}_{\mathrm{A}}$ and $\mathcal{E}_{\mathrm{B}}$. The corresponding solution is a gravitational shock-wave in $\mathrm{AdS}_{5}$, whose intersection with the boundary is the spherical shell in eq. (3.9). At generic points inside $\mathrm{AdS}_{5}$, the gravitational shock-wave involves the $\delta$-function $\delta\left(t^{2}-r^{2}-z^{2}\right)$. This will be further discussed in section 4 . 


\subsection{A time-like wave-packet}

In this subsection, we shall consider a problem which is perhaps better motivated at a physical level than the problem of the falling particle discussed in the previous subsection, but whose mathematical treatment turns out to be very similar: the decay of a time-like wave-packet. This wave-packet, which acts as a boundary condition for a supergravity field in the bulk, is to be seen as a model for the virtual photon created via $e^{+} e^{-}$annihilation. Strictly speaking, a photon within $\mathcal{N}=4$ SYM should be described by a vector field coupled to the $\mathcal{R}$-current operator, but for the present purposes there is no loss of generality if we instead consider a scalar field coupled to the 'glueball' operator $\operatorname{Tr} F_{\mu \nu}^{2}$.

Working in the rest frame of the virtual 'photon', we are led to consider the following boundary condition for the associated dilaton field in the bulk:

$$
\phi_{b}=\exp \left(-\mathrm{i} \omega t-\frac{t^{2}}{2 \sigma^{2}}-\frac{r^{2}}{2 \sigma_{r}^{2}}\right) .
$$

Clearly, this is a wave-packet with zero average momentum. ${ }^{11}$ To also guarantee that this is time-like (TL), we need to assume that both $\sigma$ and $\sigma_{r}$ are relatively large: $\sigma \omega \gg 1$ and $\sigma_{r} \omega \gg 1$. Then the Fourier modes introduced by the Gaussian in eq. (3.11) are small compared to $\omega$, which therefore sets the virtuality of the wave-packet.

Before we solve the AdS problem with boundary condition (3.11), let us first study the corresponding classical problem. In that case, the wave-packet (3.11) should be viewed as a source in the r.h.s. of the massless Klein-Gordon equation in the usual, Minkowski, space-time. The respective retarded solution reads

$$
\phi(t, \boldsymbol{r})=\int \mathrm{d} \dot{t} \mathrm{~d}^{3} \dot{\boldsymbol{r}} \Theta(t-\dot{t}) \frac{\delta(\dot{t}-t+|\boldsymbol{r}-\dot{\boldsymbol{r}}|)}{|\boldsymbol{r}-\dot{\boldsymbol{r}}|} \rho(\dot{t}, \boldsymbol{r}),
$$

where $\rho(\dot{t}, \dot{\boldsymbol{r}})$ is the same function as in eq. (3.11), but now viewed as a classical source. We are interested in the response at large distances $r \gg \sigma, \sigma_{r}$, and therefore $r \ll r$ (indeed, the Gaussian in the source sets effectively $\dot{r} \lesssim \sigma_{r}$ ). In this regime we can approximate (with $\alpha$ the angle between $\boldsymbol{r}$ and $\boldsymbol{r}$ )

$$
|\boldsymbol{r}-\dot{\boldsymbol{r}}| \simeq r-\dot{r} \cos \alpha+\frac{\dot{r}^{2}}{2 r} \sin ^{2} \alpha
$$

By keeping only the first two terms in this expansion, it is straightforward to find

$$
\phi \simeq \frac{1}{r} \exp \left[-\mathrm{i} \omega(t-r)-\frac{(t-r)^{2}}{2 \sigma^{2}}-\frac{\sigma_{r}^{2} \omega^{2}}{2}\right] .
$$

Since $\sigma_{r} \omega \gg 1$ by assumption, it is clear that this field $\phi$ is strongly suppressed (in fact, truly negligible) in the regime $r \gg \sigma_{r}$ where the above approximation is valid. This leads us to the expected conclusion that, being off-shell, a classical TL wave-packet cannot propagate outside its original support at $\dot{r} \lesssim \sigma_{r}$.

\footnotetext{
${ }^{11}$ The overall normalization of the wave-packet will be fixed later on.
} 
We now turn to the AdS calculation at strong coupling, where eq. (3.11) acts as the $4 \mathrm{D}$ boundary limit for the solution to the $5 \mathrm{D}$ Klein-Gordon equation in $\mathrm{AdS}_{5}$. Then the solution can be expressed with the help of the respective boundary to bulk propagator:

$$
\phi(t, \boldsymbol{r})=\int \mathrm{d} \dot{t} \mathrm{~d}^{3} \boldsymbol{r} \mathcal{D}(t-\dot{t}, \boldsymbol{r}-\dot{\boldsymbol{r}}, z) \phi_{b}(\dot{t}, \dot{\boldsymbol{r}}) .
$$

A convenient and rather compact expression for this propagator in coordinate space, to be derived in appendix $\mathrm{B}$, is

$$
\mathcal{D}(t-\dot{t}, \boldsymbol{r}-\dot{\boldsymbol{r}}, z)=-\frac{2 z^{4}}{\pi} \Theta(t-\dot{t}) \delta^{\prime \prime \prime}(\mathcal{W})
$$

with $\mathcal{W}$ defined in eq. (2.7). Then we can write eq. (3.15) as

$$
\phi=-\frac{z^{4}}{\pi} \partial_{z^{2}}^{3} \int \mathrm{d} \dot{t} \mathrm{~d}^{3} \dot{\boldsymbol{r}} \frac{\delta\left[\dot{t}-t+\sqrt{z^{2}+(\boldsymbol{r}-\dot{\boldsymbol{r}})^{2}}\right]}{\sqrt{z^{2}+(\boldsymbol{r}-\dot{\boldsymbol{r}})^{2}}} \phi_{b}(\dot{t}, \dot{\boldsymbol{r}}) .
$$

Once again we consider large distances $r \gg \sigma, \sigma_{r}$, where we can expand

$$
\sqrt{z^{2}+(\boldsymbol{r}-\dot{\boldsymbol{r}})^{2}} \simeq \rho-\frac{r \dot{r}}{\rho} \cos \alpha+\frac{r^{2} \dot{r}^{2}}{2 \rho^{3}} \sin ^{2} \alpha,
$$

with $\rho=\sqrt{z^{2}+r^{2}}$, and we keep the most dominant terms in the exponential. We first integrate over $\alpha$ and subsequently over $\boldsymbol{r}$, and finally we take the three derivatives on the factor which varies the most, that is $\exp (\mathrm{i} \omega \rho)$, to find

$$
\phi=\frac{\mathrm{i} \sqrt{2 \pi} \sigma_{r}^{3} \omega^{3}}{4} \frac{z^{4}}{\rho^{4}} \exp \left[-\mathrm{i} \omega(t-\rho)-\frac{(t-\rho)^{2}}{2 \sigma^{2}}-\frac{\sigma_{r}^{2} \omega^{2} r^{2}}{2 \rho^{2}}\right] .
$$

It is instructive to compare this bulk field at strong coupling to the classical field in eq. (3.14): the last term in the argument of the Gaussian in (3.19) is now proportional to $r^{2} / \rho^{2}$, and hence it can remain small even for $\sigma_{r} \omega \gg 1$ provided $r$ is sufficiently small compared to $\rho$, meaning $r \ll z$. In other terms, the wave-packet can propagate within $\mathrm{AdS}_{5}$, but only within a small angle $\theta \equiv r / z \lesssim 1 /\left(\sigma_{r} \omega\right)$ with respect to the radial axis. When $\sigma_{r} \omega \gg 1$ this angle is so small that we can neglect the displacement along the spatial direction $r$ and approximate $\rho \simeq z$. Then eq. (3.19) becomes

$$
\phi=c \exp \left[-\mathrm{i} \omega(t-z)-\frac{(t-z)^{2}}{2 \sigma^{2}}-\frac{r^{2}}{2 \theta^{2} z^{2}}\right],
$$

where $c$ is a dimensionless constant which depends on the various energy scales of our problem, but not upon the coordinates (since we have replaced $z / \rho=1$ ). At this level, it is convenient to view $c$ as a free constant, to be later fixed by the condition that the total energy carried by the TL wave-packet takes some prescribed value $E_{0}$.

In order to compute the backreaction due to this bulk wave-packet, we need the associated stress tensor. For a scalar field in $\mathrm{AdS}_{5}$ space, this is given by

$$
t_{M N}=\frac{N_{c}^{2}}{16 \pi^{2} L^{3}}\left[\partial_{M} \phi \partial_{N} \phi^{*}+\partial_{N} \phi \partial_{M} \phi^{*}-g_{M N} g^{P Q} \partial_{P} \phi \partial_{Q} \phi^{*}\right] .
$$


Under the present assumptions, we have $z \gg r \gg \sigma$. Using these conditions to simplify the components of $t_{M N}$, we eventually find

$$
t_{00}=t_{55}=-t_{05}=\frac{N_{c}^{2} c^{2} \omega^{2}}{16 \pi^{2} L^{3}} \exp \left[-\frac{(t-z)^{2}}{\sigma^{2}}-\frac{r^{2}}{\theta^{2} z^{2}}\right], \quad t_{i M}=0 .
$$

In order to arrive at these results we have dropped, in all components of $t_{M N}$, terms which are suppressed, compared to the leading non-vanishing components, by powers of $1 / \sigma \omega$ and $\sigma / z$. Recalling that $\theta \rightarrow 0$, we can replace in the above

$$
\exp \left(-\frac{r^{2}}{\theta^{2} z^{2}}\right) \simeq \pi^{3 / 2} \theta^{3} z^{3} \delta^{(3)}(\boldsymbol{r}) .
$$

Since on the other hand we have assumed $r \gg \sigma$ in order to arrive at eq. (3.22), it is clear that the use of the above $\delta$-function is legitimate only when looking at the bulk stress tensor over spatial distances much larger than $\sigma$. Remarkably, the (approximate) mathematical identity in eq. (3.23) introduces a factor $z^{3}$ in $t_{00}$, which from the manipulations in sections 2.2 and 3.1 we know to be essential in order to obtain a result for the energy density which gets contributions only from the boundary at $z=0$.

With eq. (3.23), our bulk tensor takes the form

$$
t_{00}=t_{55}=-t_{05}=E_{0} \frac{z^{3}}{L^{3}} \frac{1}{\sqrt{\pi} \sigma} \exp \left[-\frac{(t-z)^{2}}{\sigma^{2}}\right] \delta^{(3)}(\boldsymbol{r})
$$

where we have chosen the constant $c$ so that

$$
c^{2}=\frac{16 E_{0}}{N_{c}^{2} \sigma \omega^{2} \theta^{3}}=\frac{16 E_{0} \omega \sigma_{r}^{3}}{N_{c}^{2} \sigma} .
$$

It is of course understood that eq. (3.24) can be used for computing the backreaction only at sufficiently large distances $r \gg \sigma$.

As expected, eq. (3.24) is very similar to the respective expressions for the small pulse down the string, eq. (2.41), and for the falling massless particle, eq. (3.7). Once again, we have the bulk stress tensor of a source propagating along the radial direction of $\mathrm{AdS}_{5}$ at the speed of light. From our previous experience, we expect the corresponding boundary energy to be generated exclusively by the bulk field at $z=0$ and to be localized near $r=t$ within a distance $\sigma$. We have indeed $\mathcal{E}_{\mathrm{B}}=0$ and (compare to eq. (2.44))

$$
\mathcal{E}=\mathcal{E}_{\mathrm{A}}=\frac{E_{0}}{\pi^{3 / 2} \sigma} \partial_{r^{2}}^{2} \int_{0}^{\infty} \mathrm{d} z \frac{z\left(z+\sqrt{z^{2}+r^{2}}\right)}{\sqrt{z^{2}+r^{2}}} \exp \left[-\frac{\left(t-z-\sqrt{z^{2}+r^{2}}\right)^{2}}{\sigma^{2}}\right],
$$

which after manipulations entirely similar to those already encountered in section 2.2 (involving in particular the change of variable (2.45)) finally yields the following result for the energy density produced by the decay of the TL wave-packet:

$$
\mathcal{E}=\frac{E_{0}}{4 \pi r^{2}} \frac{1}{\sqrt{\pi} \sigma} \exp \left[-\frac{(t-r)^{2}}{\sigma^{2}}\right] .
$$


As anticipated this is a narrow spherical shell propagating at the speed of light, with the width set by the initial perturbation. Moreover, this energy density has been entirely produced via backreaction from the endpoint of the integration at $z=0$.

Whereas the result (3.27) shows the same type of space-time pattern as the radiation by a small string perturbation, cf. eq. (2.49), and it has been generated via similar mathematical manipulations, there is nevertheless an important difference between the two: the total energy in eq. (3.27) is independent of $\lambda$ and thus it remains constant in the strong coupling limit. This is as expected on physical grounds, since the original 'virtual photon' has only a finite energy $E_{0}$. But this raises an uncomfortable relationship between this result, eq. (3.27), and a quantum mechanical interpretation of the outgoing radiation. Namely, it seems difficult to understand how it is possible to distribute a finite amount of energy over a thin spherical shell which expands for ever (so that the energy density can become arbitrarily small) while keeping a constant, narrow, width $\sigma$ (so that the radial wave number $k_{r}$ cannot decrease below a value $k_{0} \simeq 1 / \sigma$ fixed by the uncertainty principle). Indeed, this would imply that a finite-size detector which is located sufficiently far away can register a non-zero amount of energy which is smaller than $\hbar k_{0}$, that is, smaller than the energy of a single quanta with that wave number. Classically, such a situation would be permitted, since the amplitude of the classical field corresponding to wave number $k_{r} \simeq k_{0}$ can decrease with $r$ and become arbitrarily small. But in quantum mechanics, the amplitude squared is proportional to the number of quanta per mode, which takes only discrete values.

\section{String fluctuations and the freely falling particle}

In the previous sections, we have analyzed various perturbations in the bulk associated with radiating sources on the boundary and shown that several of these perturbations (a small pulse flowing down along a string or a dilaton, time-like, wave-packet) can be mathematically idealized as a massless point particle falling freely into the radial dimension $z$ of $\mathrm{AdS}_{5}$. For all these cases, we have found that the energy flow on the boundary (i.e., in the physical space), as calculated from the backreaction of the 5D energy-momentum tensor associated with the bulk perturbation, is a thin spherical shell of matter moving outwards at the velocity of light.

Going beyond the supergravity approximation, all the bulk perturbations that were previously described by local supergravity fields should be replaced by microscopic strings. Similarly, for the problems involving a macroscopic string one should add the effects of stringy excitations. In this section, we would like to show that the world-sheet fluctuations of the microscopic strings can have sizeable effects on our previous results. To that aim, we shall take as an example the closed string underlying the 'falling point particle' introduced in section 3.1. As we shall see, depending on what measurement is made, the 'point-like particle' may be a good approximation, with string fluctuations unimportant, or a poor approximation, having important string fluctuations.

Hofmann and Maldacena [15] have already studied string fluctuations for the falling particle but only under the circumstance where energy at a given angle, or set of angles, 
is measured integrated over the three-dimensional radial coordinate. They found string corrections to angular energy-energy correlations to be small and proportional to $1 / \lambda$. However, the purpose of our study is to see whether the out-flowing energy remains in a thin radial shell when string fluctuations are included, and to carry out that calculation we need to generalize the discussion of [15].

\subsection{A special set of light-cone coordinates}

In order to describe string fluctuations, it is useful to use light-cone coordinates where the free string in flat space can be easily quantized [40,41]. Of course we are not in flat space, but so long as the fluctuations are not too large and we use coordinates where the string does not move in the fifth dimension, a flat space calculation should be adequate, at least for qualitative estimates. Clearly, our present coordinates, cf. eq. (2.1), are not well suited in that sense, since in these coordinates the 'point particle' is moving along the $z$ direction. A strategy to circumvent this problem, that was also followed in ref. [15], is to make a change of coordinates which mixes radial with boundary coordinates, in such a way that the bulk motion of our 'falling particle' occurs at a fixed value of the new fifth dimension. A suitable set of coordinates in that sense has been originally introduced in [39] and later applied to $e^{+} e^{-}$annihilation in $[15,20]$.

We shall connect these new coordinates to our previous ones by using the six global coordinates $W_{M}$ on $\mathrm{AdS}_{5}$, which satisfy

$$
W_{-1}^{2}+W_{0}^{2}-W_{1}^{2}-W_{2}^{2}-W_{3}^{2}-W_{4}^{2}=L^{2} .
$$

These are related to our previous coordinates in eq. (2.1) via

$$
W_{-1}+W_{4}=\frac{L^{2}}{z}, \quad W_{\mu}=\frac{x^{\mu} L}{z}, \quad(\mu=0,1,2,3) .
$$

In terms of them, our new coordinates, $y^{M}$, are defined as

$$
W_{0}+W_{3}=\frac{L}{y_{5}}, \quad W_{-1}=-\frac{y^{0} L}{y_{5}}, \quad W_{4}=-\frac{y^{3} L}{y_{5}}, \quad W_{1,2}=\frac{y^{1,2} L}{y_{5}},
$$

and hence they are dimensionless. In these variables, the metric (2.1) becomes

$$
\mathrm{d} s^{2}=\frac{L^{2}}{y_{5}^{2}}\left[-2 \mathrm{~d} y^{+} \mathrm{d} y^{-}+\mathrm{d} \boldsymbol{y}_{\perp}^{2}+\mathrm{d} y_{5}^{2}\right],
$$

with $y^{ \pm}=\left(y^{0} \pm y^{3}\right) / \sqrt{2}$ and $\boldsymbol{y}_{\perp}=\left(y^{1}, y^{2}\right)$. The following relations hold between the $x$ and $y$ variables (below $x^{ \pm}=\left(x^{0} \pm x^{3}\right) / \sqrt{2}$ and $\boldsymbol{x}_{\perp}=\left(x^{1}, x^{2}\right)$ )

$$
y^{+}=-\frac{L}{2 x^{+}}, \quad y^{-}=\frac{2 x^{+} x^{-}-x_{\perp}^{2}-z^{2}}{2 x^{+} L}, \quad y_{\perp}=\frac{x_{\perp}}{\sqrt{2} x^{+}}, \quad y_{5}=\frac{z}{\sqrt{2} x^{+}} .
$$

One also has $y_{5}=-\sqrt{2} y^{+} z / L$. In particular, the physical boundary at $z=0$ lies at $y_{5}=0$ in these new coordinates.

Using eq. (4.5) one can now easily check that these new variables realize indeed the desiderata for which they have been introduced: with respect to them, the bulk motion of 
the 'point particle' introduced in section 3.1 takes place at a fixed value of the (new) radial coordinate $y_{5}$. Indeed, we can write (compare to eq. (3.7))

$$
z^{3} L \delta^{(3)}(\boldsymbol{r}) \delta(z-t)=\sqrt{2} \delta\left(y_{5}-1\right) \delta^{(2)}\left(\boldsymbol{y}_{\perp}\right) \delta\left(y^{-}\right) .
$$

In these variables, the bulk excitation looks like a point-like particle propagating along the $y^{3}$ direction at the speed of light $\left(y^{3}=y^{0}\right.$, or $\left.y^{-}=0\right)$, while keeping a fixed value $y_{5}=1$ away from the boundary. It is easy to check that the only non-zero component of the particle stress tensor, eq. (3.7), in these new coordinates is ${ }^{12}$

$$
\hat{t}_{--}=\frac{E_{0}}{\sqrt{2} L^{2}} \delta\left(y_{5}-1\right) \delta^{(2)}\left(\boldsymbol{y}_{\perp}\right) \delta\left(y^{-}\right) .
$$

Note that the spherical symmetry of the problem, which was manifest in our original spatial coordinates $\boldsymbol{r}=\left(x^{1}, x^{2}, x^{3}\right)$, appears now to be lost. But this is not a serious drawback, as the full symmetry will reappear when transforming the final results for the energy density back to the original coordinates.

The new variables introduced above have another virtue, which will greatly simplify our subsequent discussion of the string fluctuations: the backreaction associated with the stress tensor (4.7) is particularly simple to compute. Indeed, with this source, the 5D system of Einstein equations reduce to a single, linear, equation for $\delta \hat{g}_{--}$which can be solved exactly, using the bulk-to-bulk propagator in $\mathrm{AdS}_{5}$. The solution is a gravitational shock-wave which at any point in the bulk is proportional to $\delta\left(y^{-}\right)$(see e.g. $[20,30,46]$ ). Then the energy-momentum tensor on the boundary is extracted in the standard way, from the behavior of $\delta \hat{g}_{--}$near $y_{5}=0$. One thus finds that the boundary response is also a shock-wave, with (below $p^{+}=E_{0} / \sqrt{2}$ )

$$
\hat{T}_{--}\left(y^{+}, y^{-}, \boldsymbol{y}_{\perp}\right)=\frac{2}{\pi} \frac{p^{+} L}{\left(1+\boldsymbol{y}_{\perp}^{2}\right)^{3}} \delta\left(y^{-}\right) .
$$

Recalling that on the boundary $\left(y_{5}=z=0\right)$ one has $y^{-} \propto 2 x^{+} x^{-}-\boldsymbol{x}_{\perp}^{2}=t^{2}-\boldsymbol{r}^{2}$, it becomes clear that what looks like a shock-wave $\left(y^{-}=0\right)$ in the $y$-coordinates is in fact the spherical shell propagating at the speed of light $(t=r)$ that we have previously found in section 3.1, cf. eq. (3.10).

This is a quite remarkable fact: the property of lack of broadening that we observed when computing the radiation in the original coordinates appears as Lorentz contraction in these new coordinates. Similarly, our observation in section 3.1, that the radiation by a massless particle falling into $\mathrm{AdS}_{5}$ comes only from $t=z \rightarrow 0$ can be viewed as a consequence of Lorentz time dilation with respect to the 'time' $y^{0}$ (or $y^{+}$). Indeed, given the discussion towards the end of section 2.1, it should be clear that the shock-wave in eq. (4.8) has been fully generated via radiation at early times $y^{+} \rightarrow-\infty$. Returning to the original variables, this implies $\dot{x}^{+} \rightarrow 0$ and therefore $\dot{t}=z \rightarrow 0$ (recall that $\dot{x}^{3}=0$

\footnotetext{
${ }^{12}$ For more clarity, we shall systematically indicate tensor components in the $y$ variables by a hat. Note also that, if one uses the coordinates $y^{0}$ and $y^{3}$ instead of the light-cone coordinates $y^{ \pm}$, then the non-zero components of $\hat{t}_{M N}$ are $\hat{t}_{00}=\hat{t}_{33}=-\hat{t}_{03}$, with $\hat{t}_{--}=2 \hat{t}_{00}$.
} 
for this source). Since the propagation at the speed of light is of course essential for such 'kinematical' arguments using the $y$ coordinates, it becomes obvious that the absence of backreaction from sources at large values of the 'original' 5 th coordinate $z$ is a consequence of the fact that these sources propagate in $\mathrm{AdS}_{5}$ at the speed of light.

Given a result like (4.8) for the (LC) energy density $\hat{T}_{--}$in the $y$ coordinates, it is useful to know how to transform it back to the original $x$ coordinates. This requires a change of variables $y^{\mu} \rightarrow x^{\mu}$ together with a Weyl transformation (see refs. [15, 20] for details). We here present the result of this transformation for the case of the energy density per unit solid angle. In the original coordinates, this is defined as

$$
\frac{\mathrm{d} E}{\mathrm{~d} \Omega}(\boldsymbol{n}) \equiv \lim _{t \rightarrow \infty} \int \mathrm{d} r r^{2} \mathcal{E}(t, \boldsymbol{r})=\lim _{r \rightarrow \infty} r^{2} \int_{0}^{\infty} \mathrm{d} t n_{i} T^{0 i}(t, r \boldsymbol{n})
$$

where the unit vector $\boldsymbol{n}=\boldsymbol{r} / r$ specifies the direction of measurement and the second equality (which is the formula used in the analysis in ref. [15]) exploits energy-momentum conservation $\left(\partial^{\mu} T_{0 \mu}=0\right)$ to express $\mathrm{d} E / \mathrm{d} \Omega$ in terms of the energy flux along $\boldsymbol{n}$ integrated over all times and evaluated at points on the sphere at $r \rightarrow \infty$. It should be clear from above formulæ that $\mathrm{d} E / \mathrm{d} \Omega$ contains no information about the radial distribution of the energy flow: this is averaged out when performing the integration over $r$, or over time.

For the case of a particle falling in $\mathrm{AdS}_{5}$, one can express either $\mathcal{E} \equiv T_{00}$ or the Poynting vector $S^{i}=T^{0 i}$ in terms of $\hat{T}_{--}$, and thus obtain $[15,20]$

$$
\frac{\mathrm{d} E}{\mathrm{~d} \Omega}(\boldsymbol{n})=\frac{\sqrt{2}\left(1+\boldsymbol{y}_{\perp}^{2}\right)^{3}}{8 L} \int \mathrm{d} y^{-} \hat{T}_{--}\left(y^{+}=0, y^{-}, \boldsymbol{y}_{\perp}\right) .
$$

In the above equation, the condition $y^{+}=0$ expresses the limit $r \rightarrow \infty$ (and hence $\left.x^{+} \rightarrow \infty\right)$. Also, $\boldsymbol{y}_{\perp}$ is related to $\boldsymbol{n}=(\sin \theta \cos \phi, \sin \theta \sin \phi, \cos \theta)$ via

$$
\boldsymbol{y}_{\perp}=\frac{\sin \theta}{1+\cos \theta}(\cos \phi, \sin \phi) \Longrightarrow \frac{2}{1+\boldsymbol{y}_{\perp}^{2}}=1+\cos \theta .
$$

(This follows from $\boldsymbol{y}_{\perp}=\boldsymbol{x}_{\perp} /\left(t+x^{3}\right)$ after using $t=r$, as appropriate for the spherical shell in eq. (4.8).) Using the above relations together with eq. (4.8) for $\hat{T}_{--}$it is straightforward to check that $\mathrm{d} E / \mathrm{d} \Omega=E_{0} / 4 \pi$, in agreement with the respective result in eq. (3.10).

\subsection{Longitudinal string fluctuations and broadening}

As shown by Hofman and Maldacena [15] the $y$ coordinates introduced in the previous subsection allow for a heuristic treatment of the world-sheet fluctuations of a microscopic string falling into $\mathrm{AdS}_{5}$ which parallels the corresponding analysis in flat space and in the light-cone gauge (see e.g. [40, 41]). In what follows we shall perform a similar analysis but focus on a different problem as compared to ref. [15]. Namely, we shall focus on the longitudinal string fluctuations $\delta y^{-}(\tau, \sigma)$, which play no role in the calculation of the angular distribution of the energy — as obvious from eq. (4.10), which involves an integral over all values of $y^{-}$— but which have the essential effect to broaden its radial distribution (in $r=|\boldsymbol{r}|$ ), as we shall see. 
Our strategy to include string fluctuations will be as follows. As previously discussed, in the absence of fluctuations the closed string reduces to a pointlike, massless, particle that in the $y$ representation is located at $y_{5}=1, \boldsymbol{y}_{\perp}=0$ and $y^{-}=0$, cf. eq. (4.7). The effect of the string fluctuations is to render this distribution 'fuzzy': a fluctuation $\delta y^{M}(\tau, \sigma)$ in the string worldsheet is responsible for a contribution to $\hat{t}_{M N}$ with support at $y_{5}=1+\delta y_{5}$, $y^{i}=\delta y^{i}$ (with $\left.i=1,2\right)$ and $y^{-}=\delta y^{-}$. There is no fluctuation in $y^{+}$by construction, because we shall work in the light-cone (LC) gauge

$$
y^{+}=\frac{\alpha^{\prime} p^{+} \tau}{L}
$$

with $p^{+}=E_{0} / \sqrt{2}$. Some general results on the quantization of string fluctuations in LC gauge and flat space are reviewed in appendix D. (The discussion in appendix D is heuristically extended here to $\mathrm{AdS}_{5}$, with $y_{5}$ treated as one of the 'transverse coordinates', on the same footing as $y^{1}$ and $y^{2}$.) As we recall in that appendix, $y^{-}(\tau, \sigma)$ is a dependent variable whose fluctuations come from those in $\boldsymbol{y}_{\perp}$ and $y_{5}$ (see eq. (D.3)). However, a given string mode only contributes $\delta y_{5} \sim \delta y^{i} \sim 1 / \lambda^{1 / 4}$ while it gives $\delta y^{-} \sim 1$ (see eqs. (D.12) and (D.13)). Moreover, the equal-point correlations ${ }^{13}\left\langle\delta y_{5}^{2}\right\rangle$ and $\left\langle\left(\delta y^{i}\right)^{2}\right\rangle$ have only weak, logarithmic, ultraviolet divergences in flat space (see eq. (D.14)) and have been argued to be finite in $\mathrm{AdS}_{5}$ [42]. By contrast, $\left\langle\left(\delta y^{-}\right)^{2}\right\rangle$ shows a strong, quadratic, UV divergence in flat space cf. eq. (D.15), thus yielding a potentially large contribution even after the introduction of an ad-hoc cutoff on the number of modes. Besides, we do not know whether one can generalize the arguments in [42] in such a way as to also cure this divergence when moving from flat space to $\mathrm{AdS}_{5}$. We shall return later to these points.

For these reasons, we henceforth neglect the fluctuations in $y^{i}$ and $y_{5}$ and concentrate on those in $y^{-}$. (The effects of $\delta y^{i}$ on the angular distribution of the energy have been estimated in ref. [15] and found to be small, of $\mathcal{O}(1 / \lambda)$.) Also, since $y^{-}=0$ for the classical pointlike particle in the bulk, cf. eq. (4.7), we denote the respective fluctuations simply as $y^{-}(\tau, \sigma)$. As usual, the acute symbol in $y^{-}$is used to differentiate a point on the string from the point $y^{-}$on the boundary at which we measure the LC energy density $\hat{T}_{--}$. Finally, in order to be able to measure the effect of the fluctuations, we need to give up the integral over $y^{-}$in eq. (4.10), but rather study the $y^{-}$-dependence of the LC energy density $\left\langle\hat{T}_{--}\right\rangle$ (averaged over the string fluctuations).

To summarize, we need to compute the backreaction from a string fluctuation with longitudinal coordinate $y^{-}(\tau, \sigma)$ and then average over the fluctuations, according to the rules in appendix D heuristically extended to $\mathrm{AdS}_{5}$. As already mentioned in the previous subsection, one advantage of the $y$ representation is that the backreaction due to the stress tensor eq. (4.7) is easy to compute. Specifically, the energy density $\hat{T}_{--}$on the boundary is obtained as the convolution of the bulk point-like source with the bulk-to-boundary

\footnotetext{
${ }^{13}$ Throughout this section, the brackets $\langle\cdots\rangle$ refer to the average over the string fluctuations.
} 
propagator (that we here take from ref. [15]). This yields

$$
\begin{aligned}
\left\langle\hat{T}_{--}\left(y^{-}, \boldsymbol{y}_{\perp}\right)\right\rangle= & \frac{6 \mathrm{i} p^{+} L}{\pi^{2}} \int_{-\infty}^{\infty} \mathrm{d} \dot{y}^{+} \int_{0}^{2 \pi} \frac{\mathrm{d} \sigma}{2 \pi} \\
& \times\left\langle\frac{\dot{y}_{5}^{4}(\tau, \sigma)}{\left[-2 \dot{y}^{+}\left(\hat{y}^{-}(\tau, \sigma)-y^{-}\right)+\dot{y}_{5}^{2}(\tau, \sigma)+\left(\boldsymbol{y}_{\perp}-\dot{y}_{\perp}(\tau, \sigma)\right)^{2}+i \epsilon\right]^{4}}\right\rangle
\end{aligned}
$$

where we shall immediately take $\dot{y}_{5}=1$ and $\dot{y}_{\perp}=0$, since we neglect the respective string fluctuations, as already explained. Also, it is here understood that $y^{+}=0$, corresponding to $x^{+} \rightarrow \infty$. At this stage it is convenient to insert unity in the form

$$
1=\int \mathrm{d} \mathfrak{y}^{-} \delta\left(\dddot{y}^{-}-\dot{y}^{-}(\tau, \sigma)\right),
$$

and thus get

$$
\left\langle\hat{T}_{--}\left(y^{-}, \boldsymbol{y}_{\perp}\right)\right\rangle=\frac{6 \mathrm{i} p^{+} L}{\pi^{2}} \int \frac{\mathrm{d} \hat{y}^{+} \mathrm{d} \hat{y}^{-}}{\left[-2 \hat{y}^{+}\left(\ddot{y}^{-}-y^{-}\right)+1+\boldsymbol{y}_{\perp}^{2}+i \epsilon\right]^{4}} P\left(\dddot{y}^{-}\right),
$$

where

$$
P\left(\dddot{y}^{-}\right) \equiv \int \frac{\mathrm{d} \sigma}{2 \pi}\left\langle\delta\left(\dddot{y}^{-}-y^{-}(\tau, \sigma)\right)\right\rangle .
$$

is recognized as the probability distribution for a point on the string to be found at $y^{-}$. In particular, it satisfies

$$
\int \mathrm{d} y^{-} P\left(\dddot{y}^{-}\right)=1
$$

as it should. Because of translation invariance we expect $P$ to be independent of $\tau$. So, the only dependence upon $y^{+}$in the integrand of eq. (4.15) is that explicit in the denominator. It is then straightforward to perform the corresponding integration, by using

$$
\int \frac{\mathrm{d} \hat{y}^{+}}{\left[-2 \hat{y}^{+}\left(\dddot{y}^{-}-y^{-}\right)+1+\boldsymbol{y}_{\perp}^{2}+i \epsilon\right]^{4}}=\frac{-\pi i}{3\left(1+\boldsymbol{y}_{\perp}^{2}\right)^{3}} \delta\left(\dddot{y}^{-}-y^{-}\right) .
$$

We thus find

$$
\left\langle\hat{\mathcal{E}}\left(y^{-}, \boldsymbol{y}_{\perp}\right)\right\rangle \equiv \frac{\sqrt{2}\left(1+\boldsymbol{y}_{\perp}^{2}\right)^{3}}{8 L}\left\langle\hat{T}_{--}\left(y^{-}, \boldsymbol{y}_{\perp}\right)\right\rangle=\frac{E_{0}}{4 \pi} P\left(y^{-}\right) .
$$

The new quantity $\left\langle\hat{\mathcal{E}}\left(y^{-}, \boldsymbol{y}_{\perp}\right)\right\rangle$ is defined in such a way that its integral over $y^{-}$yields the energy density per unit angle, cf. eq. (4.10). By integrating the last expression above over $y^{-}$and using the probability conservation (4.17), one recovers the previous result $\mathrm{d} E / \mathrm{d} \Omega=E_{0} / 4 \pi$. This meets our expectations: the effects of the longitudinal string fluctuations are washed out by the integral over $y^{-}$.

But the crucial new feature in eq. (4.19) is its explicit dependence upon $y^{-}$. Note the remarkable fact that the integral over $y^{+}$in eq. (4.18) has identified the longitudinal coordinate of a point on the string $\left(y^{-}\right)$with that of a point on the boundary $\left(y^{-}\right)$. In 
the absence of fluctuations, $P\left(y^{-}\right) \rightarrow \delta\left(y^{-}\right)$, and the above expression yields, as expected, the shock-wave result in eq. (4.8) - that is, a spherical shell of zero width $(t=r)$ in the original $x$ coordinates. But for generic fluctuations, eq. (4.19) shows the interesting result that the width of the probability distribution $P\left(y^{-}\right)$for the fluctuations acts at the same time as the width of the distribution in $y^{-}$of the energy density on the boundary. This is the announced relation between fluctuations and broadening.

To better appreciate the physical meaning of this relation, it is useful to recall that the relation between the coordinates $y^{M}$ and the original coordinates $x^{M}$ looks different in the bulk $(z>0)$ as compared to the boundary $(z=0)$.

The argument $y^{-}$of $P\left(y^{-}\right)$refers to a point on the closed string in the bulk. Using eq. (4.5), we can relate string fluctuations in $y^{-}$to fluctuations in the position $\dot{x}^{M}$ of the 'massless falling particle' in $\mathrm{AdS}_{5}$. (For more clarity, we reintroduce the 'acute' symbol on coordinates belonging to the string and the $\delta$ symbol for fluctuations: e.g. $y^{-} \rightarrow \delta y^{-}$.) In doing that, one should recall that the variable $\dot{x}^{+}=-L /\left(2 \hat{y}^{+}\right)$is by construction fixed in the LC gauge (4.12). Then, one can separate the average motion of the falling particle $\left(z_{0}=\sqrt{2} \dot{x}^{+}, \boldsymbol{r}_{0}=0\right)$ from its fluctuations by rewriting eq. (4.5) as

$$
\begin{gathered}
z=\sqrt{2} \dot{x}^{+}\left(1+\delta y_{5}\right), \quad \dot{x}_{\perp}=\sqrt{2} \dot{x}^{+} \delta \dot{y}_{\perp}, \\
\delta \dot{y}^{-}=-\frac{1}{L}\left[\sqrt{2} \delta \dot{x}^{3}+\dot{x}^{+}\left(\delta \dot{y}_{\perp}^{2}+\delta y_{5}^{2}+2 \delta y_{5}\right)\right] .
\end{gathered}
$$

The first two equations above show that fluctuations in $z$ and $\dot{x}_{\perp}$ are of the same order as those in the 'transverse coordinates' $y_{5}$ and $\dot{\boldsymbol{y}}_{\perp}$, and hence they are suppressed at large $\lambda$. As for the last equation, it shows that, in physical coordinates, the large string fluctuations in $y^{-}$correspond to fluctuations in the spatial coordinate $\dot{x}^{3}: \delta \dot{x}^{3} \sim L \delta \dot{y}^{-}$. This lack of spherical symmetry for the problem at hand might look surprising at first sight, but it is just an artifact of our change of variables (4.5) together with the use of the LC gauge. But this has no incidence on the final result for the energy density in physical space, which is obtained by combining (4.10) and (4.19) and is manifestly isotropic.

Returning to eq. (4.19), the argument $y^{-}$of $\left\langle\hat{T}_{--}\right\rangle$is a boundary variable, for which $z=0$ and hence

$$
y^{-}=\frac{2 x^{+} x^{-}-x_{\perp}^{2}}{2 x^{+} L}=\frac{t-r}{\sqrt{2} L} \quad \text { for } \quad x_{\perp}=0,
$$

where in the second equality we have used the underlying spherical symmetry to fix $\boldsymbol{x}_{\perp}=0$ (and hence $r=x^{3}$ ). Hence, via eq. (4.19), the large string fluctuations in $y^{-}$are directly mapped onto fluctuations $\Delta r$ in the localization of the energy distribution around a central value $r=t$. Specifically, if the probability distribution $P\left(y^{-}\right)$for the string fluctuations has some characteristic width $\Delta y^{-}$, then (4.19) and (4.21) imply that there will be a width

$$
\Delta r=L \Delta y^{-}
$$

for the radial "energy shell" on the boundary at time $t$.

This broadening of the energy distribution is in agreement with the UV/IR correspondence, as we now explain. To that aim, we return to the integral representation (4.15) but, 
instead of performing the integral over $y^{+}$as in eq. (4.18), we consider first the integral over $\dddot{y}^{-}$. If the probability distribution $P\left(\dddot{y}^{-}\right)$has support in a range $\Delta \hat{y}^{-}$, then the integral over $\dddot{y}^{-}$within that range requires $\left|\dot{y}^{+}\right| \lesssim 1 / \Delta \hat{y}^{-}$. But from eq. (4.5), $-2 \dot{y}^{+}=L / \dot{x}^{+}$ so that $\dot{x}^{+} \sim L \Delta \dot{y}^{-}$. For the falling particle $\dot{x}^{+}=z / \sqrt{2}$, so we finally get the following estimate

$$
z \sim L \Delta y^{-}
$$

for the typical values of $z$ contributing to fluctuations with amplitude $\Delta y^{-}$. By comparing with eq. (4.22), we see that $\Delta r \sim z$, which is the expected correlation according to the $\mathrm{UV} / \mathrm{IR}$ correspondence. Note also that this correlation is washed out - once again, as expected - when integrating the energy distribution over $y^{-}$. Indeed, if one integrates eq. (4.15) over $y^{-}$, by using a formula analogous to eq. (4.18), one generates the $\delta$-function $\delta\left(\dot{y}^{+}\right)$, which then implies $\dot{x}^{+}=z / \sqrt{2} \rightarrow \infty$. Thus, the angular energy distribution, as obtained after integration the radial distribution over $r$ (or $t$ ), appears to be generated via backreaction at $z \rightarrow \infty$, as already noted in ref. [15].

Finally, how large is the width $\Delta y^{-}$of the probability distribution $P\left(y^{-}\right)$for the string fluctuations? This can be identified with the squared root of $\left\langle\left(\delta y^{-}\right)^{2}\right\rangle$, which is the equalpoint limit of the respective 2-point function. In flat space at least, $\left\langle\left(\delta y^{-}\right)^{2}\right\rangle$ shows a strong UV divergence, so the above question makes no sense without a cutoff on the number of contributing string modes. Similar, and even stronger, UV problems are also inherent in the evaluation of $P\left(\dddot{y}^{-}\right)$according to eq. (4.16): by exponentiating the $\delta$-function in the integrand there and then expanding the exponential, one generates an infinite series of higher-point correlations of $y^{-}$, which are evaluated at coincident points. To obtain an heuristic estimate for $\Delta y^{-}$, let us introduce an upper limit $N$ on the mode number $n$ for the string oscillations. Requiring $n \leq N$, one finds (see appendix D)

$$
\left\langle\left(\Delta y^{-}\right)^{2}\right\rangle \sim \frac{N^{2}}{\left(L p^{+}\right)^{2}},
$$

which shows a much stronger cutoff dependence than the respective transverse fluctuations

$$
\left\langle\left(\Delta y^{i}\right)^{2}\right\rangle \sim \frac{\ln N}{\sqrt{\lambda}} .
$$

If, as argued in [42], the warping in the 5th dimension acts effectively as a UV cutoff in $\mathrm{AdS}_{5}$, then it seems natural to take $N$ of the order

$$
N \sim e^{\sqrt{\lambda}}
$$

since via eq. (4.25) this yields a result for $\left\langle\left(\Delta y^{i}\right)^{2}\right\rangle$ which is finite and independent of $\lambda$, in agreement with [42]. With this choice for $N$, eqs. (4.24) and (4.22) yield a very large spread in $r$ away from $r=t$. As noticed after eq. (4.19), the space-time structure of this spread is in principle determined by the probability distribution $P\left(y^{-}\right)$for longitudinal string fluctuations. But in the absence of a reliable procedure for computing string fluctuations in $\mathrm{AdS}_{5}$, we are not able to specify this distribution any further. 
Of course, in reality we do not have a good control over string fluctuations when they become so large as shown in (4.24)-(4.26). However, we do believe that we have given good arguments for the broadening of the radial profile of the outgoing energy produced by the decay of a time-like photon. Moreover, it appears that the scale $\Delta y^{-}$which controls this broadening according to (4.22) is large and not suppressed by inverse powers of $\lambda$. This implies strong modifications of the predictions of the supergravity approximation, whose accurate calculation would require a proper treatment of string fluctuations in $\mathrm{AdS}_{5}$.

\section{Acknowledgments}

During the late stages of this work, three of us (E. I., A.H. M., and D.N. T.) have benefited from financial support from the Erwin Schrödinger International Institute for Mathematical Physics in Vienna, within the context of the ESI Programme on AdS Holography and the Quark-Gluon Plasma (Vienna, 2 August-29 October 2010), organized by A. Rebhan, S. Husa, and K. Landsteiner. We would like to thank ESI and the organizers of the Programme for their hospitality and for offering us most suitable conditions for work and for intense and fruitful discussions. In particular, during our stay in Vienna we have enjoyed and benefited from discussions with J. Casalderrey-Solana, P. Chesler, E. Kiritsis, I. Klebanov, R. Myers, D. Teaney, and A. Yarom. We thank J. Casalderrey-Solana, G. Giecold and U. Gürsoy for useful comments on the manuscript. The work of Y. H. is supported by Special Coordination Funds for Promoting Science and Technology of the Ministry of Education, the Japanese Government. The work of E. I. is supported in part by Agence Nationale de la Recherche via the programme ANR-06-BLAN-0285-01. The work of A.H. M. is supported in part by the US Department of Energy.

\section{A The constant velocity string: a different perspective}

In this appendix we present an alternative way to derive the energy energy density induced by a heavy quark moving with constant velocity. Considering eqs. (2.12) and (2.13), we make the change of variables

$$
\dot{t}=t_{q}+\gamma z \Rightarrow \mathrm{d} \dot{t}=\mathrm{d} t_{q}
$$

and then $\mathcal{W}$ becomes a linear function of $z$, more precisely

$$
\mathcal{W}=\mathcal{W}_{q}+2 \gamma z\left(t-v x-\frac{t_{q}}{\gamma^{2}}\right)
$$

where we have defined

$$
\mathcal{W}_{q}=-\left(t-t_{q}\right)^{2}+\left(x-v t_{q}\right)^{2}+\boldsymbol{x}_{\perp}^{2}
$$

Now it is straightforward to integrate over $z$ making use of the $\delta$-function which will set

$$
z=-\frac{\mathcal{W}_{q}}{2 \gamma\left(t-v x-t_{q} / \gamma^{2}\right)}
$$


Causality requires that the denominator in the above eq. (A.4) should be positive and, since $z \geq 0$, we need $W_{q} \leq 0$ in order to obtain a non-zero result. Thus we have

$$
\mathcal{E}_{\mathrm{A}}=\frac{\sqrt{\lambda}}{2 \pi^{2}} \gamma^{2}\left(3-v^{2}\right) \partial_{\boldsymbol{x}_{\perp}^{2}}^{2} \int_{-\infty}^{\infty} \mathrm{d} t_{q} \frac{\Theta\left(-\mathcal{W}_{q}\right)}{\gamma^{2}(t-v x)-t_{q}} .
$$

It is clear that the first derivative will act on the step function, that is, on the upper end point of the integration interval, to give

$$
\partial_{\boldsymbol{x}_{\perp}^{2}} \Theta\left(-\mathcal{W}_{q}\right)=-\delta\left(\mathcal{W}_{q}\right)=-\frac{\gamma \delta\left[t_{q}-\gamma^{2}(t-v x)+\gamma \sqrt{\boldsymbol{x}_{\perp}^{2}+\gamma^{2}(x-v t)^{2}}\right]}{2 \sqrt{\boldsymbol{x}_{\perp}^{2}+\gamma^{2}(x-v t)^{2}}} .
$$

Now also the integration over $t_{q}$ can be easily done by making use of the $\delta$-function in the above. We find

$$
\mathcal{E}_{\mathrm{A}}=-\frac{\sqrt{\lambda}}{4 \pi^{2}} \gamma^{2}\left(3-v^{2}\right) \partial_{\boldsymbol{x}_{\perp}^{2}} \frac{1}{\boldsymbol{x}_{\perp}^{2}+\gamma^{2}(x-v t)^{2}}
$$

which leads to eq. (2.16) in the main text, and similarly we can calculate $\mathcal{E}_{\mathrm{B}}$ which leads to eq. (2.18). What is important to notice in the above derivation, is that the $t_{q}$-integration is determined by $\mathcal{W}_{q}=0$, which in turn fixes $z=0$ when performing the $z$-integration.

\section{B Boundary to bulk scalar propagator in $\mathrm{AdS}_{5}$}

Here we would like to construct the boundary to bulk scalar propagator. We shall obtain it from the $-z^{4} / 4$ coefficient of the bulk to bulk propagator. In momentum space, and for space-like kinematics, the latter is given by (see for example appendix B in [38])

$$
G(\omega, \boldsymbol{q}, z, \dot{z})=-z_{<}^{2} z_{>}^{2} \mathrm{I}_{2}\left(Q z_{<}\right) \mathrm{K}_{2}\left(Q z_{>}\right),
$$

where $Q^{2}=\boldsymbol{q}^{2}-\omega^{2}>0$. In the time-like region $Q^{2}<0$ the propagator is obtained by analytic continuation together with the retardation prescription $\omega \rightarrow \omega+\mathrm{i} 0$. Then we find for the boundary to bulk propagator

$$
D(\omega, \boldsymbol{q}, z)= \begin{cases}\frac{z^{2} Q^{2}}{2} \mathrm{~K}_{2}(Q z) & \text { if } \quad Q^{2}>0, \\ \frac{\mathrm{i} \pi z^{2}|Q|^{2}}{4} \mathrm{H}_{2}^{(1)}(|Q| z) & \text { if } \quad Q^{2}<0, \omega>0, \\ -\frac{\mathrm{i} \pi z^{2}|Q|^{2}}{4} \mathrm{H}_{2}^{(2)}(|Q| z) & \text { if } \quad Q^{2}<0, \omega<0 .\end{cases}
$$

Notice that $D(\omega, \boldsymbol{q}, z=0)=1$. Now we wish to Fourier transform back to configuration space. Performing the angular integrations in spherical coordinates we find

$$
D(\omega, \boldsymbol{r}, z)=\int \frac{\mathrm{d}^{3} \boldsymbol{q}}{(2 \pi)^{3}} \mathrm{e}^{\mathrm{i} \boldsymbol{q} \cdot \boldsymbol{r}} D(\omega, \boldsymbol{q}, z)=\frac{1}{2 \pi^{2} r} \int_{0}^{\infty} \mathrm{d} q q \sin (q r) D(\omega, \boldsymbol{q}, z)
$$


with $r=|\boldsymbol{r}|$ and $q=|\boldsymbol{q}|$. For $\omega>0$ we can use the first two cases in eq. (B.2) and integrating over $q$ we obtain

$$
D(\omega>0, \boldsymbol{r}, z)=\frac{\mathrm{i}}{8 \sqrt{2 \pi}} \frac{z^{4}}{\rho^{7 / 2}} \omega^{7 / 2} \mathrm{H}_{7 / 2}^{(1)}(\omega \rho),
$$

where we have defined $\rho=\sqrt{r^{2}+z^{2}}$. We notice that $\mathrm{H}_{7 / 2}$ is a rather simple function which for $\omega>0$ is given by

$$
\mathrm{H}_{7 / 2}^{(1)}(\omega \rho)=\sqrt{\frac{2}{\pi \omega \rho}} \mathrm{e}^{\mathrm{i} \omega \rho}\left[1-\frac{6}{\mathrm{i} \omega \rho}+\frac{15}{(\mathrm{i} \omega \rho)^{2}}-\frac{15}{(\mathrm{i} \omega \rho)^{3}}\right] .
$$

For $\omega<0$ we have

$$
D(\omega<0, \boldsymbol{r}, z)=D^{*}(|\omega|, \boldsymbol{r}, z),
$$

and therefore we can write

$$
D(t, \boldsymbol{r}, z)=2 \int \frac{\mathrm{d} \omega}{2 \pi} \operatorname{Re}\left[\mathrm{e}^{-\mathrm{i} \omega t} D(\omega, \boldsymbol{r}, z)\right]
$$

Making use of eqs. (B.4) and (B.5) we see that the integrand in the above is an even function of $\omega$ and therefore we can multiply by a factor of $1 / 2$ and extend the integration to the whole real axis. Furthermore the terms odd in $\omega$ will vanish automatically and the result will be real. Thus

$$
D(t, \boldsymbol{r}, z)=-\frac{z^{4}}{8 \pi \rho^{4}} \int_{-\infty}^{\infty} \frac{\mathrm{d} \omega}{2 \pi}(\mathrm{i} \omega)^{3} \mathrm{e}^{\mathrm{i} \omega(\rho-t)}\left[1-\frac{6}{\mathrm{i} \omega \rho}+\frac{15}{(\mathrm{i} \omega \rho)^{2}}-\frac{15}{(\mathrm{i} \omega \rho)^{3}}\right] .
$$

The last expression is simply related to an integral representation of the $\delta$-function and its derivatives and we have

$$
D(t, \boldsymbol{r}, z)=-\frac{z^{4}}{8 \pi \rho^{4}}\left[\delta^{\prime \prime \prime}(\rho-t)-\frac{6 \delta^{\prime \prime}(\rho-t)}{\rho}+\frac{15 \delta^{\prime}(\rho-t)}{\rho^{2}}-\frac{15 \delta(\rho-t)}{\rho^{3}}\right] .
$$

It is a straightforward exercise to start from $16 \rho^{4} \Theta(t) \delta^{\prime \prime \prime}\left(\rho^{2}-t^{2}\right)$ and show that it is equal to the square bracket above (in the sense of distributions). Thus we finally have

$$
D(t, \boldsymbol{r}, z)=-\frac{2 z^{4}}{\pi} \Theta(t) \delta^{\prime \prime \prime}\left(\rho^{2}-t^{2}\right) .
$$

\section{Backreaction in the non-relativistic limit}

Let us add here some complemental details in the calculation to the energy radiated by a non-relativistic quark when it goes under an arbitrary 1D motion. The string embedding functions and their derivatives are given by

$$
X^{M}=\left(t, x_{s}, 0,0, z\right), \quad \dot{X}^{M}=\left(1, \dot{x_{s}}, 0,0,0\right), \quad X^{M^{\prime}}=\left(0, x_{s}^{\prime}, 0,0,1\right)
$$


and we easily find the components and the determinant of the induced metric on the world-sheet (with the usual parametrization $(\tau, \sigma) \equiv(t, z)$ )

$$
\begin{aligned}
g_{\tau \tau} & =\dot{X} \cdot \dot{X}=\left|G_{00}\right|\left(-1+\dot{x}_{s}^{2}\right), \\
g_{\sigma \sigma} & =X^{\prime} \cdot X^{\prime}=\left|G_{00}\right|\left(1+x_{s}^{\prime 2}\right), \\
g_{\tau \sigma} & =\dot{X} \cdot X^{\prime}=\left|G_{00}\right| \dot{x}_{s} x_{s}^{\prime}, \\
\sqrt{-g} & =\left|G_{00}\right| \sqrt{1-\dot{x}_{s}^{2}+x_{s}^{\prime 2}} .
\end{aligned}
$$

Now starting from eq. (2.9) we can find

$$
\tilde{t}^{M N}=\frac{T_{0}}{\sqrt{-g} \sqrt{-G}}\left[g_{\sigma \sigma} \dot{X}^{M} \dot{X}^{N}+g_{\tau \tau} X^{M^{\prime}} X^{N^{\prime}}-g_{\tau \sigma}\left(\dot{X}^{M} X^{N^{\prime}}+X^{M^{\prime}} \dot{X}^{N}\right)\right] .
$$

Let us calculate the components of the above expression and at the same time lower the indices. Since the metric is diagonal we do this by multiplying each component by $\pm\left|G_{00}\right|^{2}$, where we use the minus only when one of the two indices is equal to 0. After substitution of the common coefficient

$$
\frac{T_{0}\left|G_{00}\right|^{3}}{\sqrt{-g} \sqrt{-G}}=\frac{\sqrt{\lambda}}{2 \pi} \frac{z}{L^{3}} \frac{1}{\sqrt{1-\dot{x}_{s}^{2}+x_{s}^{\prime 2}}},
$$

we find

$$
\begin{array}{rlrl}
\tilde{t}_{00} & =\frac{\sqrt{\lambda}}{2 \pi} \frac{z}{L^{3}} \frac{1+x_{s}^{\prime 2}}{\sqrt{1-\dot{x}_{s}^{2}+x_{s}^{\prime 2}}}, & \tilde{t}_{01} & =-\frac{\sqrt{\lambda}}{2 \pi} \frac{z}{L^{3}} \frac{\dot{x}_{s}}{\sqrt{1-\dot{x}_{s}^{2}+x_{s}^{\prime 2}}}, \\
\tilde{t}_{05}=\frac{\sqrt{\lambda}}{2 \pi} \frac{z}{L^{3}} \frac{\dot{x}_{s} x_{s}^{\prime}}{\sqrt{1-\dot{x}_{s}^{2}+x_{s}^{\prime 2}}}, & \tilde{t}_{11}=\frac{\sqrt{\lambda}}{2 \pi} \frac{z}{L^{3}} \frac{\dot{x}_{s}^{2}-x_{s}^{\prime 2}}{\sqrt{1-\dot{x}_{s}^{2}+x_{s}^{\prime 2}}}, \\
\tilde{t}_{15}=-\frac{\sqrt{\lambda}}{2 \pi} \frac{z}{L^{3}} \frac{x_{s}^{\prime}}{\sqrt{1-\dot{x}_{s}^{2}+x_{s}^{\prime 2}}}, & \tilde{t}_{55}=\frac{\sqrt{\lambda}}{2 \pi} \frac{z}{L^{3}} \frac{-1+\dot{x}_{s}^{2}}{\sqrt{1-\dot{x}_{s}^{2}+x_{s}^{\prime 2}}} .
\end{array}
$$

Expanding the square root and keeping only the quadratic terms of the components we have

$$
\tilde{t}_{00}=\tilde{t}_{55}=\frac{\sqrt{\lambda}}{4 \pi} \frac{z}{L^{3}}\left(\dot{x}_{s}^{2}+x_{s}^{\prime 2}\right), \quad \tilde{t}_{05}=\frac{\sqrt{\lambda}}{2 \pi} \frac{z}{L^{3}} \dot{x}_{s} x_{s}^{\prime}, \quad \tilde{t}_{11}=\frac{\sqrt{\lambda}}{2 \pi} \frac{z}{L^{3}}\left(\dot{x}_{s}^{2}-x_{s}^{\prime 2}\right)
$$

and using eq. (2.37) to express $\dot{x}_{s}$ and $x_{s}^{\prime}$ in terms of the boundary motion we finally arrive at eq. (2.41). We should notice here that $\tilde{t}_{01}$ and $\tilde{t}_{15}$ contain a linear term in $\dot{x}_{s}$ and $x_{s}^{\prime}$ respectively. However, they do not contain a term quadratic in the boundary motion since the first correction to the solution to the string EOM eq. (2.27) is cubic in the boundary motion. Indeed, one can check by direct substitution that

$$
x_{s}(t, z)=x_{q}(t-z)+z \dot{x}_{q}(t-z)-\frac{1}{2} z^{2} \dot{x}_{q}^{2}(t-z) \ddot{x}_{q}(t-z),
$$

is the solution to the required order of accuracy. But in general, one might consider the situation where terms in $t_{M N}$ which are constant or linear in $\dot{x}_{s}$ and $x_{s}^{\prime}$ combine with the expansion of $\delta(\mathcal{W})$ in powers of $x_{s}$ and therefore produce quadratic terms which can give 
rise to radiation (recall that we have set $x_{s}=0$ in the argument of $\mathcal{W}$ in section 2.2). However one can find that

$$
\delta(\mathcal{W})=\frac{1}{2 \rho} \delta(\dot{t}-t+\rho)+\frac{x x_{s}}{2 \rho^{3}}\left[\delta(\dot{t}-t+\rho)-\rho \delta^{\prime}(\dot{t}-t+\rho)\right]+\mathcal{O}\left(x_{s}^{2}\right),
$$

with $\rho=\sqrt{z^{2}+r^{2}}$, and such terms will be eventually suppressed by inverse power of $r$ and thus do not contribute to the radiated energy.

\section{Strings in the light-cone gauge}

Here we remind the reader how the free string in flat space is quantized and evaluate the size of the fluctuations (for more details see e.g. [40, 41]). As usual, we denote the worldsheet coordinates by $\tau$ and $\sigma$, with $0 \leq \sigma \leq 2 \pi$ for a closed string. We shall work in the light-cone (LC) gauge, defined by

$$
y^{+}=\frac{\alpha^{\prime} p^{+} \tau}{L}
$$

$\left(p^{+}\right.$denotes the LC momentum of the string) together with the associated $\sigma$ parametrization. In this gauge, the transverse coordinates $\boldsymbol{y}_{\perp}(\tau, \sigma)=\left(y^{1}, y^{2}\right)$ of the string obey the equations of motion of harmonic oscillators,

$$
\left(\frac{\partial^{2}}{\partial \tau^{2}}-\frac{\partial^{2}}{\partial \sigma^{2}}\right) y^{i}(\tau, \sigma)=0
$$

whereas the longitudinal coordinate $y^{-}$is not independent, but rather it is related to the transverse coordinates by the constraints enforcing the LC gauge. Namely, one has (for a closed string)

$$
\partial_{\tau} y^{-}=\frac{L}{2 \alpha^{\prime} p^{+}}\left(\left(\partial_{\tau} \boldsymbol{y}_{\perp}\right)^{2}+\left(\partial_{\sigma} \boldsymbol{y}_{\perp}\right)^{2}\right) .
$$

eq. (D.2) is solved as (below, $i=1,2$ )

$$
y^{i}(\tau, \sigma)=y_{0}^{i}+\sqrt{\frac{\alpha^{\prime}}{2 L^{2}}}\left(\alpha_{0}^{i}+\tilde{\alpha}_{0}^{i}\right) \tau+\mathrm{i} \sqrt{\frac{\alpha^{\prime}}{2 L^{2}}} \sum_{n \neq 0}\left\{\frac{\alpha_{n}^{i}}{n} \mathrm{e}^{-\mathrm{i} n(\sigma+\tau)}+\frac{\tilde{\alpha}_{n}^{i}}{n} \mathrm{e}^{\mathrm{i} n(\sigma-\tau)}\right\} .
$$

The $\alpha$ operators with/without a tilde refer to closed string waves which are left/right moving along the string. The quantization of the transverse string fluctuations proceeds in the standard way, by imposing

$$
\left[\alpha_{m}^{i}, \alpha_{n}^{j}\right]=m \delta^{i j} \delta_{m,-n},
$$

together with the similar relation for the tilded operators. (Tilded and untilded operators commute with each other.) Notice that the standard creation and annihilation operators for the quantum harmonic oscillator are related to the $\alpha_{n}^{i}$ operators above via

$$
a_{n}^{i}=\frac{\alpha_{n}^{i}}{\sqrt{n}}, \quad a_{n}^{i \dagger}=\frac{\alpha_{-n}^{i}}{\sqrt{n}}, \quad \text { with } \quad n \geq 1
$$


and obey indeed $\left[a_{n}^{i}, a_{m}^{i \dagger}\right]=\delta^{i j} \delta_{m n}$.

The constraint equation (D.3) can be integrated to yield

$$
y^{-}(\tau, \sigma)=y_{0}^{-}+\frac{\tau}{2 p^{+} L}\left(L_{0}^{\perp}+\tilde{L}_{0}^{\perp}\right)+\frac{\mathrm{i}}{2 p^{+} L} \sum_{n \neq 0} \frac{1}{n}\left(L_{n}^{\perp} \mathrm{e}^{-\mathrm{i} n(\sigma+\tau)}+\tilde{L}_{n}^{\perp} \mathrm{e}^{\mathrm{i} n(\sigma-\tau)}\right),
$$

where

$$
L_{n}^{\perp}=\frac{1}{2} \sum_{p} \alpha_{n-p}^{i} \alpha_{p}^{i}, \quad L_{-n}^{\perp}=\left(L_{n}^{\perp}\right)^{\dagger}
$$

are the so-called transverse Virasoro generators which satisfy the commutation relation

$$
\left[L_{m}, L_{n}\right]=(m-n) L_{m+n}+\frac{c}{12}\left(m^{3}-m\right) \delta_{m,-n} .
$$

$c$ is the central charge which equals the number of the transverse dimensions $D-2$.

The two-point functions of these operators are evaluated in the standard way:

$$
\begin{aligned}
\left\langle y^{i}(\tau, \sigma), y^{j}(0,0)\right\rangle & =\delta^{i j} \frac{\alpha^{\prime}}{2 L^{2}} \sum_{n=1}^{\infty} \frac{1}{n}\left(\mathrm{e}^{-i n(\sigma+\tau)}+\mathrm{e}^{i n(\sigma-\tau)}\right), \\
\left\langle y^{-}(\tau, \sigma) y^{-}(0,0)\right\rangle & =\frac{c}{48\left(p^{+} L\right)^{2}} \sum_{n=1}^{\infty} \frac{n^{2}-1}{n}\left(\mathrm{e}^{-i n(\sigma+\tau)}+\mathrm{e}^{i n(\sigma-\tau)}\right) .
\end{aligned}
$$

From eq. (D.10) it is clear that the size of the transverse fluctuation $\delta y^{i}$ due to a single mode $n$ is

$$
\delta y_{n}^{i} \sim \sqrt{\frac{\alpha^{\prime}}{n L^{2}}}=\frac{1}{\sqrt{n \sqrt{\lambda}}},
$$

which is very small when $\lambda$ is large. On the other hand, eq. (D.11) implies that a single mode gives a longitudinal fluctuation with typical size

$$
\delta y_{n}^{-} \sim \frac{1}{p^{+} L}
$$

which is not suppressed when $\lambda$ is large and which gets much larger contributions from large $n$ as compared to $\delta y^{i}$. This last feature becomes especially important whenever one needs to consider the equal-point limit of the 2-point function (or, more generally, of a $n$-point function), so like in the discussion of the average LC energy $\langle\hat{\mathcal{E}}\rangle$ in section 4.2.

Specifically, when taking the limit $\tau, \sigma \rightarrow 0$ in eqs. (D.10) and (D.11) one encounters ultraviolet divergences coming from the sum over the large $-n$ modes, which are logarithmic in the case of eq. (D.10), but quadratic in the case of eq. (D.11). Introducing a mode cutoff $n \leq N$ to regularize the divergence, we get

$$
\begin{aligned}
\left\langle\left(y^{i}\right)^{2}\right\rangle & =\frac{\alpha^{\prime}}{L^{2}} \ln N=\frac{\ln N}{\sqrt{\lambda}}, \\
\left\langle\left(y^{-}\right)^{2}\right\rangle & =\frac{c N^{2}}{48\left(p^{+} L\right)^{2}} .
\end{aligned}
$$


What should be the value of the mode cutoff $N$ ? If we were to consider a scattering problem - the scattering between two strings - then, first, longitudinal modes would not matter and, second, the effective region in $\tau$ would be non-zero and fixed by the kinematics: $\delta \tau \sim 1 /\left(\alpha^{\prime} s\right)$ with $\sqrt{s}$ the center of mass energy of the scattering. This limits the participating modes to $n \leq N=\alpha^{\prime} s$ and the corresponding fluctuation sizes to

$$
\left\langle\left(y^{i}\right)^{2}\right\rangle \sim \frac{\ln \alpha^{\prime} s}{\sqrt{\lambda}}, \quad\left\langle\left(y^{-}\right)^{2}\right\rangle \sim \frac{\left(\alpha^{\prime} s\right)^{2}}{\left(p^{+} L\right)^{2}} .
$$

The first equation in (D.16) gives the shrinkage of the diffraction peak while the second one gives the natural longitudinal extent of the scattering process.

However, when evaluating $\langle\hat{\mathcal{E}}\rangle$ in eq. (4.13) or (4.15), one encounters higher powers of $y^{-}(\tau, \sigma)$ which must be averaged and there is no natural cutoff. Polchinski and Susskind [42] argue that in $\mathrm{AdS}_{5}$ the string fluctuations are cutoff by warping in the fifth dimension, in such a way that (D.14) becomes finite and of order one (and thus independent of $\sqrt{\lambda}$ ). This suggests

$$
N \sim \mathrm{e}^{\sqrt{\lambda}}
$$

as a cutoff on modes which in turn would give, cf. eq. (D.15),

$$
\Delta y^{-} \sim \frac{e^{\sqrt{\lambda}}}{p^{+} L}
$$

which is a very large value. Note that

$$
x \sim e^{-\sqrt{\lambda}}
$$

is the region in Bjorken- $x$ variable where one starts to see "partons" in DIS at strong coupling [36]. Then

$$
\Delta y^{-} \sim \frac{1}{x p^{+}},
$$

can be identified with the lifetime of partons which have energy $x p^{+}-$here the partons which compose the closed string, or the 'falling point particle'.

Open Access. This article is distributed under the terms of the Creative Commons Attribution Noncommercial License which permits any noncommercial use, distribution, and reproduction in any medium, provided the original author(s) and source are credited.

\section{References}

[1] J.M. Maldacena, The large- $N$ limit of superconformal field theories and supergravity, Int. J. Theor. Phys. 38 (1999) 1113 [Adv. Theor. Math. Phys. 2 (1998) 231] [hep-th/9711200] [SPIRES].

[2] S.S. Gubser, I.R. Klebanov and A.M. Polyakov, Gauge theory correlators from non-critical string theory, Phys. Lett. B 428 (1998) 105 [hep-th/9802109] [SPIRES]. 
[3] E. Witten, Anti-de Sitter space and holography, Adv. Theor. Math. Phys. 2 (1998) 253 [hep-th/9802150] [SPIRES].

[4] D.T. Son and A.O. Starinets, Viscosity, Black Holes and Quantum Field Theory, Ann. Rev. Nucl. Part. Sci. 57 (2007) 95 [arXiv: 0704.0240] [SPIRES].

[5] E. Iancu, Partons and jets in a strongly-coupled plasma from AdS/CFT, Acta Phys. Polon. B 39 (2008) 3213 [arXiv:0812.0500] [SPIRES].

[6] S.S. Gubser, S.S. Pufu, F.D. Rocha and A. Yarom, Energy loss in a strongly coupled thermal medium and the gauge-string duality, arXiv:0902.4041 [SPIRES].

[7] A. Mikhailov, Nonlinear waves in AdS/CFT correspondence, hep-th/0305196 [SPIRES].

[8] C.P. Herzog, A. Karch, P. Kovtun, C. Kozcaz and L.G. Yaffe, Energy loss of a heavy quark moving through $N=4$ supersymmetric Yang-Mills plasma, JHEP 07 (2006) 013 [hep-th/0605158] [SPIRES].

[9] S.S. Gubser, Drag force in AdS/CFT, Phys. Rev. D 74 (2006) 126005 [hep-th/0605182] [SPIRES].

[10] J. Casalderrey-Solana and D. Teaney, Heavy quark diffusion in strongly coupled $N=4$ Yang-Mills, Phys. Rev. D 74 (2006) 085012 [hep-ph/0605199] [SPIRES].

[11] S.S. Gubser, Momentum fluctuations of heavy quarks in the gauge-string duality, Nucl. Phys. B 790 (2008) 175 [hep-th/0612143] [SPIRES].

[12] J. Casalderrey-Solana and D. Teaney, Transverse momentum broadening of a fast quark in a $N=4$ Yang-Mills plasma, JHEP 04 (2007) 039 [hep-th/0701123] [SPIRES].

[13] S.S. Gubser, S.S. Pufu and A. Yarom, Energy disturbances due to a moving quark from gauge- string duality, JHEP 09 (2007) 108 [arXiv:0706.0213] [SPIRES].

[14] P.M. Chesler and L.G. Yaffe, The wake of a quark moving through a strongly-coupled $\mathcal{N}=4$ supersymmetric Yang-Mills plasma, Phys. Rev. Lett. 99 (2007) 152001 [arXiv:0706.0368] [SPIRES].

[15] D.M. Hofman and J. Maldacena, Conformal collider physics: Energy and charge correlations, JHEP 05 (2008) 012 [arXiv: 0803.1467] [SPIRES].

[16] Y. Hatta, E. Iancu and A.H. Mueller, Jet evolution in the $N=4$ SYM plasma at strong coupling, JHEP 05 (2008) 037 [arXiv: 0803.2481] [SPIRES].

[17] S.S. Gubser, D.R. Gulotta, S.S. Pufu and F.D. Rocha, Gluon energy loss in the gauge-string duality, JHEP 10 (2008) 052 [arXiv:0803.1470] [SPIRES].

[18] P.M. Chesler, K. Jensen and A. Karch, Jets in strongly-coupled $N=4$ super Yang-Mills theory, Phys. Rev. D 79 (2009) 025021 [arXiv:0804.3110] [SPIRES].

[19] F. Dominguez, C. Marquet, A.H. Mueller, B. Wu and B.-W. Xiao, Comparing energy loss and $p_{\perp}$-broadening in perturbative $Q C D$ with strong coupling $\mathcal{N}=4 S Y M$ theory, Nucl. Phys. A 811 (2008) 197 [arXiv:0803.3234] [SPIRES].

[20] Y. Hatta, Relating $e^{+} e^{-}$annihilation to high energy scattering at weak and strong coupling, JHEP 11 (2008) 057 [arXiv:0810.0889] [SPIRES].

[21] K.B. Fadafan, H. Liu, K. Rajagopal and U.A. Wiedemann, Stirring Strongly Coupled Plasma, Eur. Phys. J. C 61 (2009) 553 [arXiv:0809.2869] [SPIRES]. 
[22] M. Chernicoff and A. Guijosa, Acceleration, Energy Loss and Screening in Strongly-Coupled Gauge Theories, JHEP 06 (2008) 005 [arXiv: 0803.3070] [SPIRES].

[23] C. Athanasiou, P.M. Chesler, H. Liu, D. Nickel and K. Rajagopal, Synchrotron radiation in strongly coupled conformal field theories, Phys. Rev. D 81 (2010) 126001 [arXiv:1001.3880] [SPIRES].

[24] P. Arnold and D. Vaman, Jet quenching in hot strongly coupled gauge theories revisited: 3-point correlators with gauge-gravity duality, JHEP 10 (2010) 099 [arXiv: 1008.4023] [SPIRES].

[25] L. Susskind and E. Witten, The holographic bound in anti-de Sitter space, hep-th/9805114 [SPIRES].

[26] A.W. Peet and J. Polchinski, UV/IR relations in AdS dynamics, Phys. Rev. D 59 (1999) 065011 [hep-th/9809022] [SPIRES].

[27] Y. Hatta, E. Iancu, A.H. Mueller and D.N. Triantafyllopoulos, in preparation.

[28] C.G. Callan Jr. and A. Guijosa, Undulating strings and gauge theory waves, Nucl. Phys. B 565 (2000) 157 [hep-th/9906153] [SPIRES].

[29] V.E. Hubeny, Relativistic Beaming in AdS/CFT, arXiv:1011.1270 [SPIRES].

[30] S.S. Gubser, S.S. Pufu and A. Yarom, Entropy production in collisions of gravitational shock waves and of heavy ions, Phys. Rev. D 78 (2008) 066014 [arXiv:0805.1551] [SPIRES].

[31] S.S. Gubser, I.R. Klebanov and A.M. Polyakov, A semi-classical limit of the gauge/string correspondence, Nucl. Phys. B 636 (2002) 99 [hep-th/0204051] [SPIRES].

[32] A.V. Kotikov, L.N. Lipatov, A.I. Onishchenko and V.N. Velizhanin, Three-loop universal anomalous dimension of the Wilson operators in $N=4$ SUSY Yang-Mills model, Phys. Lett. B 595 (2004) 521 [hep-th/0404092] [SPIRES].

[33] R.C. Brower, J. Polchinski, M.J. Strassler and C.-I. Tan, The Pomeron and Gauge/String Duality, JHEP 12 (2007) 005 [hep-th/0603115] [SPIRES].

[34] Y. Hatta and T. Matsuo, Jet fragmentation and gauge/string duality, Phys. Lett. B 670 (2008) 150 [arXiv:0804.4733] [SPIRES].

[35] J. Polchinski and M.J. Strassler, Deep inelastic scattering and gauge/string duality, JHEP 05 (2003) 012 [hep-th/0209211] [SPIRES].

[36] Y. Hatta, E. Iancu and A.H. Mueller, Deep inelastic scattering at strong coupling from gauge/string duality: the saturation line, JHEP 01 (2008) 026 [arXiv:0710.2148] [SPIRES].

[37] Y. Hatta, E. Iancu and A.H. Mueller, Deep inelastic scattering off a $N=4$ SYM plasma at strong coupling, JHEP 01 (2008) 063 [arXiv:0710.5297] [SPIRES].

[38] E. Avsar, E. Iancu, L. McLerran and D.N. Triantafyllopoulos, Shockwaves and deep inelastic scattering within the gauge/gravity duality, JHEP 11 (2009) 105 [arXiv:0907.4604] [SPIRES].

[39] L. Cornalba, Eikonal Methods in AdS/CFT:Regge Theory and Multi-Reggeon Exchange, arXiv:0710.5480 [SPIRES].

[40] J. Polchinski, String theory. Vol. 1: An introduction to the bosonic string, Cambridge University Press, Cambridge U.K. (1998). 
[41] B. Zwiebach, A first course in string theory, Cambridge University Press, Cambridge U.K. (2004).

[42] J. Polchinski and L. Susskind, String theory and the size of hadrons, hep-th/0112204 [SPIRES].

[43] S. de Haro, S.N. Solodukhin and K. Skenderis, Holographic reconstruction of spacetime and renormalization in the AdS/CFT correspondence, Commun. Math. Phys. 217 (2001) 595 [hep-th/0002230] [SPIRES].

[44] K. Skenderis, Lecture notes on holographic renormalization, Class. Quant. Grav. 19 (2002) 5849 [hep-th/0209067] [SPIRES].

[45] P.M. Chesler and L.G. Yaffe, The stress-energy tensor of a quark moving through a strongly-coupled $N=4$ supersymmetric Yang-Mills plasma: comparing hydrodynamics and AdS/CFT, Phys. Rev. D78 (2008) 045013 [arXiv:0712.0050] [SPIRES].

[46] S.S. Gubser, S.S. Pufu and A. Yarom, Off-center collisions in AdS $S_{5}$ with applications to multiplicity estimates in heavy-ion collisions, JHEP 11 (2009) 050 [arXiv:0902.4062] [SPIRES]. 